\title{
GUILHERME TAMANINI
}

\begin{abstract}
Análise da confiabilidade do posicionamento dos eletrodos na aquisição do sinal eletromiográfico dos músculos do antebraço na tarefa de preensão palmar
\end{abstract}

Versão Corrigida

Dissertação apresentada ao Programa de Pós-Graduação em Reabilitação e Desempenho Funcional da Faculdade de Medicina de Ribeirão Preto da Universidade de São Paulo para a obtenção do título de Mestre.

Orientadora: $\operatorname{Prof}^{\mathrm{a}} \mathrm{Dr}^{\mathrm{a}}$ Marisa de Cássia Registro Fonseca

Ribeirão Preto 
Autorizo a reprodução e divulgação total ou parcial deste trabalho, por qualquer meio convencional ou eletrônico, para fins de estudo e pesquisa, desde que citada a fonte.

Tamanini, Guilherme

Análise da confiabilidade do posicionamento dos eletrodos na aquisição do sinal eletromiográfico dos músculos do antebraço na tarefa de preensão palmar. Ribeirão Preto, 2015.

69 p.: il. ; $30 \mathrm{~cm}$

Dissertação de Mestrado, apresentada à Faculdade de Medicina de Ribeirão Preto/USP. Área de concentração: Reabilitação e Desempenho Funcional

Orientador: Fonseca, Marisa de Cássia Registro 
TAMANINI, Guilherme

Análise da confiabilidade do posicionamento dos eletrodos na aquisição do sinal eletromiográfico dos músculos do antebraço na tarefa de preensão palmar.

Dissertação apresentada à Faculdade de Medicina de Ribeirão Preto da Universidade de São Paulo para obtenção do título de mestre.

Aprovado em:

Banca Examinadora

Prof.(a) Dr. (a) Instituição:

Julgamento: Assinatura:

Prof.(a) Dr. (a) Instituição:

Julgamento: Assinatura:

Prof.(a) Dr. (a) Instituição:

Julgamento: Assinatura: 
Aos meus pais, irmãos, a minha família e a minha esposa Rosângela. 


\section{AGRADECIMENTOS}

Agradeço a Deus onde nos momentos mais difíceis me deu serenidade para continuar.

Aos meus pais, por tudo que me deram e me proporcionaram.

Aos meus irmãos e irmãs (cunhadas) que estão sempre ao meu lado me incentivando e me apoiando em tudo. São minhas inspirações.

À minha amada esposa, Rosângela, pois sem você nada disso teria acontecido. Sempre ao meu lado, sofrendo junto e comemorando junto todas as conquistas. Você é tudo.

À Prof ${ }^{a}$. Dr ${ }^{\mathrm{a}}$. Marisa pela oportunidade e pela confiança em mim, de que um dia eu poderia realizar e conquistar mais uma etapa da minha vida profissional. Você foi imprencidível para essa realização.

Ao Prof. Dr. Alexandre pelos conselhos e parceria nestes anos. Você me ensinou muito.

Ao Prof. Dr. Rafael por ter me incentivado em mais esta etapa e por, a mim, confiar e colaborar em seu doutorado.

A Eula pelos ensinamentos, rizadas, parceria, por tudo, você me ajudou muito neste processo. Em você me espelhei várias vezes.

A todos do LabMão, pela colaboração e pela amizade.

Ao pessoal do LAPOMH, Tennysson e Jaqueline, por compartilharem seus conhecimentos e espaço para a realização deste projeto.

Aos fisioterapeutas do CER, Daniel, Larissa, Raquel, Aline e Amira por permitirem que uma etapa deste processo fosse realizada.

Ao Prof. Dr. Mazzer por disponibilizar o ambulatório para recrutamento. 
Aos aprimorandos que auxiliaram no recrutamento dos voluntários.

Aos amigos e parceiros do Hospital Estadual pelos incentivos e pelos ombros amigos nos momentos difíceis.

Ao Prof. Edson que me deu a oportunidade de colocar em prática todo o ensinamento que tive neste trabalho.

A todos do Centro Universitário Claretiano por me receberem de braços abertos.

Aos meus amigos/irmãos Victor, Daniel, Grassi, Thalita e Karol por me incentivarem a iniciar no mestrado e pelos ensinamentos.

Ao Samuel pelo apoio acadêmico.

Aos amigos e mestrandos Marília Zanin, Gabriel, Cesário e Harumi por trilharem estes mesmos caminhos compartilhando as expectativas, inseguranças, fraquezas e sucessos.

A todos que colaboraram de alguma forma para a realização deste estudo.

Aos voluntários pela participação no estudo. 


\section{RESUMO}

Tamanini, G. Análise da confiabilidade do posicionamento dos eletrodos na aquisição do sinal eletromiográfico dos músculos do antebraço na tarefa de preensão palmar. 2015. 69f. Dissertação (Mestrado) - Faculdade de Medicina de Ribeirão Preto, Universidade de São Paulo, Ribeirão Preto, 2015.

O objetivo deste estudo foi de analisar a confiabilidade teste reteste do posicionamento dos eletrodos na aquisição do sinal eletromiográfico dos músculos do antebraço na tarefa de preensão palmar, por meio de dois posicionamentos. $O$ primeiro posicionamento proposto por Cram; Durie e o segundo proposto por Mogk; Keir. Foram recrutados 30 voluntários sendo divididos em 2 grupos com 15 indivíduos cada. O primeiro grupo era formado por indivíduos saudáveis, isto é, que não apresentavam qualquer patologia, trauma ou dor no membro superior. Este grupo era composto por 13 mulheres e 2 homens com idade média de 23,4 anos, sendo 13 destros e 2 canhotos. O segundo grupo era formado por indivíduos que apresentavam algum trauma no antebraço, punho ou mão, recrutados no centro de reabilitação e no ambulatório de cirurgia do membro superior do Hospital das Clínicas da Faculdade de Medicina de Ribeirão Preto da Universidade de São Paulo. A amostra era composta por 13 homens e 2 mulheres com idade média de 34,6 anos, sendo 14 destros e 1 canhoto. Todos os participantes assinaram o Termo de Consentimento Livre e Esclarecido. O procedimento foi realizado em dois dias com diferença de $24 \mathrm{~h}$ entre as avaliações. A avaliação consistia em realizar a tarefa de preensão palmar com o dinamômetro analógico da marca Jamar $^{\circledR}$, realizando a máxima contração voluntária pelo período de 10 segundos com repouso de 3 minutos entre cada preensão, sendo coletados o sinal eletromiográfico de sete canais dos músculos do antebraço volar e dorsal, segundo cada método. Foi analisada a confiabilidade pelo índice de ICC e o SEM (Standard Error of Measurement). Os resultados obtidos mostram excelente correlação entre os dois posicionamentos em ambos os grupos. Quando verificado o valor de ICC do grupo de saudáveis verificou valores acima de 0.75 em todos os canais, o que demonstrou uma alta correlação entre as medidas em ambos os posicionamentos, com pequeno SEM. No grupo pacientes foi observado que nos músculos FUC, FSD e ERC uma correlação moderada para o posicionamento Mogk; Keir e somente no músculo FRC apresentou correlação moderada no posicionamento Cram; Durie. Os gráficos de 
Bland e Altman mostraram alta concordância entre as medidas, visto que, em ambos os posicionamentos, os valores ficaram entre o intervalo de confiança de $95 \%$ e próximos da linha média.

Como conclusão pode-se inferir que ambos os posicionamentos apresentaram alta confiabilidade e alta correlação quando avaliado por meio do teste e re-teste, podendo ser utilizados para medida eletromiográfica dos músculos do antebraço.

Palavras-chave: Eletromiografia, antebraço, confiabilidade 


\begin{abstract}
Tamanini, G. Reliability of the positioning of the electrodes in the acquisition of electromyography activity in forearm muscles in the hand grip task. 2015. 69f. Master Dissertation - Ribeirao Preto Medicine School, University of São Paulo, Ribeirao Preto, 2015.
\end{abstract}

The objective of this study was to analyze test retest reliability of the electrodes placement in the acquisition of electromyography forearm muscles during a isometric hand grip task, through two different methods. The first method was proposed by Cram; Durie and the second by Mogk; Keir. We recruited 30 volunteers divided into 2 groups with 15 subjects each. The first group consisted of healthy subjects, who did not show any pathology, trauma or pain in the upper extremity. This group consisted of 13 women and 2 men with a mean age of 23.4 years, and 13 right-handed and 2 left-handed. The second group consisted of individuals who had previous trauma on the forearm, wrist or hand. They were recruited from the rehabilitation center and upper limb surgery clinic of the Medical School of Ribeirão Preto Clinical Hospital. The sample consisted of 13 men and 2 women with a mean age of 34.6 years, 14 right-handed and left-handed one. All participants signed the consent form. The procedure was performed in two days with 24 hours difference between assessments. The evaluation was to carry by a isometric handgrip task with a analog dynamometer Jamar®, with maximum voluntary contraction for 10 seconds with 3 minutes of rest and analised by $\mathrm{CCl}$ and SEM (Standard Error of Measurement). The results obtained have a high correlation between the two methods in both groups. Analyzing the ICC value of the healthy group value noted above 0.75 in all channels, demonstrating a excelent correlation between the measurements in both positions and low SEM. Checking in group patients realize that the FUC, FSD and ERC muscles has a moderate correlation for Mogk; Keir positioning and only on FRC showed moderate correlation in Cram; Durie electrode placement method. Analyzing Bland and Altman method a high concordance was 
found between the measures, since in both positions, the values were between the confidence interval of $95 \%$ and close to the midline. Through this we can infer that both positions have high reliability and high correlation when measured by the test and retest.

Keywords: Electromyography, forearm, reliability 


\section{LISTA DE FIGURAS}

Figura 1 - Posicionamento do sujeito e material utilizado durante a tarefa........

Figura 2 - Disposição dos eletrodos segundo método de Mogk e Keir (ano 2003)

Figura 03 - Gráfico de dispersão de concordância entre medidas de BlandAltman no método Mogk do canal 01 na captação eletromiográfica nos modos teste e re-teste em ambos os grupos

Figura 4 - Gráfico de dispersão de concordância entre medidas de Bland-

Altman no método Mogk do canal 02 na captação eletromiográfica nos modos teste e re-teste em ambos os grupos

Figura 05 - Gráfico de dispersão de concordância entre medidas de BlandAltman no método Mogk do canal 03 na captação eletromiográfica nos modos teste e re-teste ambos os grupos

Figura 06 - Gráfico de dispersão de concordância entre medidas de Bland-

Altman no método Mogk do canal 04 na captação eletromiográfica nos modos teste e re-teste em ambos os grupos

Figura 07 - Gráfico de dispersão de concordância entre medidas de BlandAltman no método Mogk do canal 05 na captação eletromiográfica nos modos teste e re-teste em ambos os grupos

Figura 08 - Gráfico de dispersão de concordância entre medidas de BlandAltman no método Mogk do canal 06 na captação eletromiográfica nos modos teste e re-teste em ambos os grupos

Figura 09 - Gráfico de dispersão de concordância entre medidas de BlandAltman no método Mogk do canal 07 na captação eletromiográfica nos modos teste e re-teste em ambos os grupos

Figura 10 - Gráfico de dispersão de concordância entre medidas de BlandAltman no método Cram do canal 01 na captação eletromiográfica nos modos teste e re-teste em ambos os grupos

Figura 11 - Gráfico de dispersão de concordância entre medidas de BlandAltman no método Cram do canal 02 na captação eletromiográfica nos modos teste e re-teste em ambos os grupos 
Figura 12 - Gráfico de dispersão de concordância entre medidas de BlandAltman no método Cram do canal 03 na captação eletromiográfica nos modos teste e re-teste em ambos os grupos

Figura 13 - Gráfico de dispersão de concordância entre medidas de BlandAltman no método Cram do canal 04 na captação eletromiográfica nos modos teste e re-teste em ambos os grupos

Figura 14 - Gráfico de dispersão de concordância entre medidas de BlandAltman no método Cram do canal 05 na captação eletromiográfica nos modos teste e re-teste em ambos os grupos

Figura 15 - Gráfico de dispersão de concordância entre medidas de BlandAltman no método Cram do canal 06 na captação eletromiográfica nos modos teste e re-teste em ambos os grupos

Figura 16 - Gráfico de dispersão de concordância entre medidas de BlandAltman no método Cram do canal 07 na captação eletromiográfica nos modos teste e re-teste em ambos os grupos 


\section{LISTA DE TABELAS}

Tabela 01 - Teste de força muscular segundo Kendall et. al. (2005)

Tabela 02 - Média e Desvio Padrão dos valores de RMS normalizados dos grupos de pacientes e não pacientes, obtidos no teste e re-teste dos posicionamentos Mogk e Cram

Tabela 03 - Média e Desvio Padrão dos valores de RMS normalizados do grupo não pacientes, obtidos no teste e re-teste dos posicionamentos Mogk e Cram

Tabela 04 - Média e Desvio Padrão dos valores de RMS normalizados do grupo pacientes, obtidos no teste e re-teste dos posicionamentos Mogk e Cram

Tabela 05 - Valores do coeficiente de correlação intra-classe (ICC) para confiabilidade intra-dia e erro padrão de medida (SEM) referente aos valores de RMS normalizados de ambos os grupos

Tabela 06 - Valores do coeficiente de correlação intra-classe (ICC) para confiabilidade intra-dia e erro padrão de medida (SEM) referente aos valores de RMS normalizados do grupo pacientes

Tabela 07 - Valores do coeficiente de correlação intra-classe (ICC) para confiabilidade intra-dia e erro padrão de medida (SEM) referente aos valores de RMS normalizados do grupo não pacientes. 


\section{LISTA DE ABREVIAÇÕES E SIGLA}

EMG Eletromiografia

SENIAM Surface Electromyography for the Non-Invasive Assessment of Muscles sEMG Eletromiografia de Superfície

AVD's Atividades de Vida Diária

ICC Coeficiente de Correlação Intra-classe

CVM Contração Voluntária Máxima

RMS Root Means Square

SEM Erro Padrão de Medida 


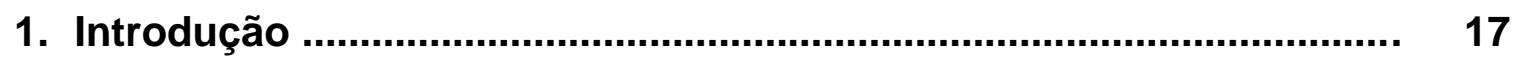

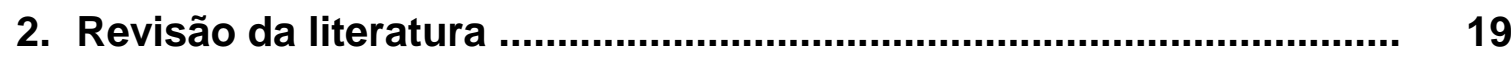

2.1. Anatomia e Função dos Músculos do Antebraço.............................. $\quad 20$

2.2. Eletromiografia ..................................................................... 22

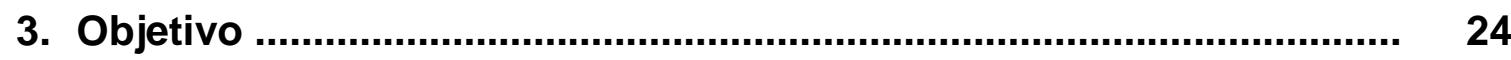

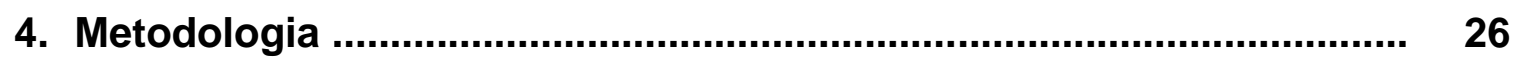

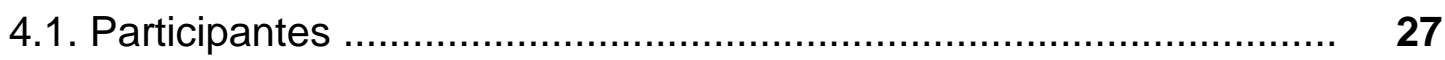

4.2. Posicionamento corporal durante os testes .................................. $\quad 28$

4.3. Posicionamento dos eletrodos .................................................. $\quad 28$

4.4. Normalização do sinal .......................................................... $\mathbf{3 1}$

4.5. Procedimento de registro eletromiográfico ................................... $\quad 31$

4.6 Análise Estatística.................................................................. 31

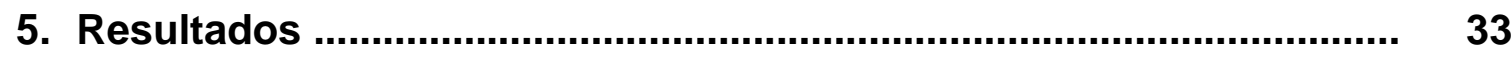

5.1. Valores de Amplitude Eletromiográfica - RMS ............................. $\quad 34$

5.2. Valores de confiabilidade ............................................................ 36

5.3. Gráfico de Dispersão de Bland-Altman .......................................... $\quad 38$

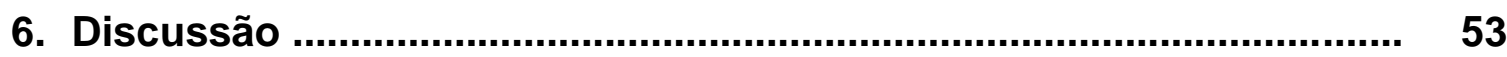

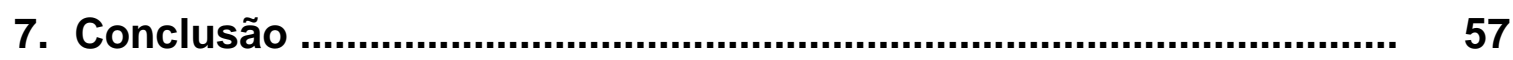

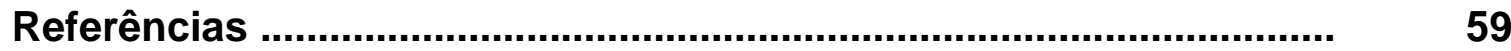

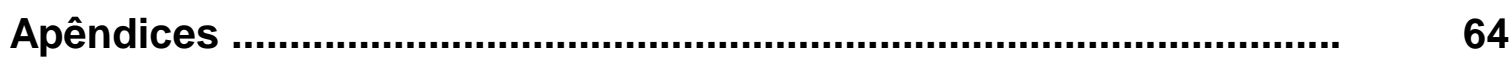

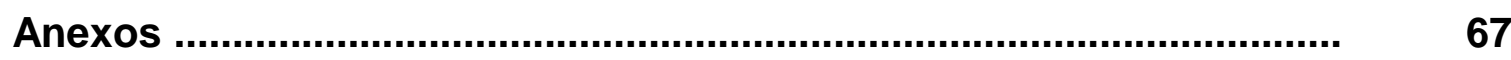




\section{INTRODUÇÃO}




\section{INTRODUÇÃO}

A eletromiografia de superfície é um importante instrumento para a avaliação da função muscular através da investigação dos sinais elétricos que surgem quando o músculo é ativado (DE LUCA, 1997).

Nas últimas décadas houve um grande aumento do conhecimento dessa técnica (HERMENS, et al., 2000). Porém, com diferentes tipos de metodologias, as quais tornavam difícil a sua reprodutibilidade. Com isso ficou evidente que era necessária uma padronização dos componentes da eletromiografia para maior integralidade entre os estudos e assim consequente maior confiabilidade da ferramenta (HERMENS et. al., 2000).

Em 1996, um grupo de pesquisadores europeus formulou uma serie de recomendações para o uso da eletromiografia. Este grupo recomendou algumas padronizações para a aquisição do sinal EMG, tais como: tipos de sensores, colocação dos sensores, posicionamento dos eletrodos, preparo da pele, processamento do sinal e modulação (HERMENS et. al., 2000). A partir de tais recomendações foi criado o SENIAM (Surface Electromyography for the NonInvasive Assessment of Muscles).

A padronização da colocação dos eletrodos pelo corpo humano, ainda apresenta lacunas a serem analisadas, entre elas os músculos da região do antebraço. Este seguimento corporal apresenta as origens e inserções musculares muito próximas uma das outras e os ventres musculares pequenos. Com isso, diferenças no posicionamento dos eletrodos e do membro durante as coletas apresentaram métodos diferentes e assim de difícil padronização (HERMENS et. al., 2000).

A relação eletrodo de superfície e o músculo a ser avaliado, sofre interferências, o que exige o máximo de atenção do avaliador no momento do posicionamento dos eletrodos. Tal fato ocorre de maneira substancial no antebraço. (MOGK; KEIR, 2003). 
Esta dificuldade técnica pode ser um dos motivos que tem levado a poucos estudos na literatura sobre uma metodologia reprodutível sobre o posicionamento dos eletrodos na musculatura do antebraço.

Muito disso deve-se a dificuldade em se isolar a musculatura durante a aquisição, apresentando cruzamentos entre os sinais captados dos músculos tornando infidedigno o sinal coletado (MOGK; KEIR, 2003; OSKOUEI; PAULIN; CARMAN, 2013).

O "Crosstalk", que é o sinal detectado sobre um músculo, porém devido a proximidade entre os ventres musculares e assim as unidades motoras, os eletrodos de superfície captam o sinal gerado por um outro músculo, fazendo com que tal procedimento se torne um erro (FARINA et. al., 2004).

Estes sinais cruzados estão relacionados aos eletrodos de eletromiografia de superfície (sEMG). Outro tipo de eletrodo, denominado de agulha, se mostra mais eficaz quando se trata de diminuir o "Crosstalk" (FINSEN et. al., 2005).

Para isso estudos de confiabilidade se tornam necessários. Confiabilidade é a propriedade do instrumento de alcançar um escore onde o erro é mínimo. Toda técnica de medição apresenta um valor de erro, e quando este erro é muito pequeno, em comparação ao valor verdadeiro, os resultados daquele instrumento serão confiáveis (FAGARASANU; KUMAR, 2002; DE VET, et al. 2011). Um instrumento, para ser confiável, necessita que o mesmo avaliador e outros avaliadores possam reproduzir e utilizar o instrumento que os resultados obtidos terão o mesmo valor, o que torna o instrumento fidedigno (MACDERMID, 2011). 
REVISÃO DA LITERATURA 


\section{REVISÃO DA LITERATURA}

\subsection{Anatomia e Função dos Músculos do Antebraço}

O ser humano para realizar uma atividade, seja qual ela for, recreativa ou laboral, precisa ou grossa, necessita de coordenação motora e propriocepção. No corpo humano o membro superior é capaz de realizar todas essas atividades com maestria como Rosén \& Lundborg (2007) afirmou em seu estudo relatando que a mão é um instrumento delicado do indivíduo, sendo o elo com o ambiente.

Para que isso ocorra de maneira sincronizada e coordenada a mão apresenta diversos pequenos músculos que apresentam origem e inserção nela própria, sendo chamados de músculos intrínsecos. Porém, a mão necessita de um maior número de músculos para realizar todas as atividades que ela é capaz de realizar, então músculos originados no antebraço e cotovelo são necessários para tal fato. Estes músculos são denominados extrínsecos em relação a mão.

O antebraço apresenta uma composição de 14 músculos com uma secção transversal média de $30 \mathrm{~cm}$, como o extensor curto e longo do carpo, extensor ulnar do carpo, extensor comum dos dedos, extensor próprio do indicador, extensor próprio do dedo mínimo, o supinador, flexor radial do carpo, flexor ulnar do carpo, palmar longo, pronador redondo, flexor superficial e profundo dos dedos, com origem comum nos epicôndilos do úmero, transpondo o cotovelo e indo se inserir nos ossos do antebraço-rádio e ulna e também nos ossos da mão (NEUMANN, 2002).

Quando o indivíduo apresenta a ligação entre a mão e o cérebro preservada, com um sistema proprioceptivo e tátil em condições normais e com o mecanismo antecipatório integro, isto faz com que apresente uma boa preensão (ROSÉN; LUNDBORG, 2007; HERMSDÖRFER et. al., 2011). E para isso ocorrer os músculos possuem mecanismos fisiológicos que permitem o movimento (CRAM; DURIE, 2011). 
Apresentando tais condições o antebraço se mostra com um importante papel na função de fornecer um suporte forte e estável para que ocorra um melhor posicionamento da mão no espaço e assim poder realizar a tarefa de preensão (ZIMMERMAN, 2002).

Como pode-se verificar no estudo de Strickland (2005) que relata que como em todas as articulações do corpo humano a mão necessita que ocorra um perfeito sinergismo entre a musculatura extrínseca/intrínseca e flexora/extensora para que haja um adequado movimento, seja ele de precisão ou de força.

$\mathrm{Na}$ preensão é necessária uma combinação entre o polegar e os outros dedos, sendo o anular e o mínimo os mais importantes para a realização do movimento. O polegar é o responsável por envolver o objeto no plano contrário aos dedos, sendo tal movimento chamado de oponência (STRICKLAND, 2005).

Os músculos flexores extrínsecos dos dedos proporcionam a principal força para preensão. $O$ extensor comum dos dedos fornece estabilidade aos flexores. Os interósseos rodam a primeira falange e flexionam a articulação metacarpofalangeana. Com exceção do quarto, os lumbricais não participam da preensão palmar. Já os músculos tenares e o adutor do polegar proporcionam força de compressão contra o objeto. Portanto, a posição de maior preensão palmar é a posição funcional do punho caracterizada pela amplitude entre $10^{\circ}$ de flexão e $35^{\circ}$ de extensão segundo (NEUMANN, 2002).

O uso da preensão consiste em um movimento de abrir a mão, envolver o objeto com os dedos e o polegar, estender o punho à $30^{\circ}$ e realizar a volta da extensão da metacarpofalangiana e interfalangianas proximal e distal dos dedos e realizar a volta da abdução do polegar. Para que essa tarefa ocorra são necessários os músculos extensor comum dos dedos, extensor próprio do indicador, extensor próprio do dedo mínimo, extensor radial longo e curto do carpo, lumbricais e abdutor curto do polegar (NEUMANN, 2002; FESS, 2011).

Portanto, qualquer alteração no sistema musculoesquelético do antebraço irá alterar a função da mão em realizar as atividades de vida diária (AVD's), atividade laboral e esportiva, como escrever, virar cartas, arremessar, manipular pequenos objetos, comer e movimentar objetos grandes, sendo eles leves e/ou pesados (POREMBA; JARUS, 1993; STRICKLAND, 2005; RIORDAN, 1995). 


\subsection{Eletromiografia}

A eletromiografia fornece acesso aos processos fisiológicos da contração muscular, na qual irá gerar uma força, realizará um movimento e finalizar com incontáveis funções. (DE LUCA, 1997).

No membro superior uma destas funções é a preensão e a eletromiografia é um instrumento com uma parte importante na avaliação e quantificação da qualidade desta contração muscular. Porém há uma ausência de estudos que verifiquem a confiabilidade desta ferramenta nas avaliações do antebraço, punho e mão (OSKOUEI; PAULIN; CARMAN, 2013).

Em outras estruturas corporais há o inverso, com uma grande quantidade de estudos que mostram a confiabilidade da ferramenta, avaliando diferentes tipos de exercícios (ARAÚJO et. al., 2009), tipos de eletrodos (HAIG et. al., 2003), posicionamento de eletrodos (HERMENS et. al., 2000), métodos de aquisição de sinal (OLLIVIER et. al., 2005) e avaliação nas tarefas ergonômicas (BAO; SILVERSTEIN, 2005).

Como no estudo de Araújo et. al. (2009) com a qual objetivava avaliar a confiabilidade intra-dias e inter-dias nos valores de amplitude da eletromiografia dos músculos do braço e tronco em 3 diferentes tipos de exercícios. A conclusão do estudo revelou uma ótima confiabilidade quando realizados no mesmo dia em relação a diferentes dias.

Um fato no estudo de $\mathrm{Ng}$ et. al. (2003) que chama atenção é que a quantidade de força realizada pode ser um fator que afete a confiabilidade, isto é, quando se avalia a força máxima há um consequente aumento da variabilidade no sinal eletromiográfico.

Concomitante a este fato, a literatura nos mostra que não há diferença entre os valores do coeficiente de correlação intra-classe (ICC) e o erro padrão de medida quando avaliados entre o 50\% da contração voluntária máxima (CVM) e 100\% da CVM (OSKOUEI; PAULIN; CARMAN, 2013). 
Uma outra forma de se aumentar a confiabilidade de uma ferramenta é realizar a média das medidas de repetições daquela tarefa a ser analisada e realizar a aquisição de grupos musculares ao invés de individuais. Isto faz com que o Crosstalk seja amenizado, principalmente no antebraço (OSKOUEI; PAULIN; CARMAN, 2013; IACONO, 2004).

Porém, o estudo de Mogk; Keir, (2003) que avalia tal interferência em tarefas realizadas com a rotação do antebraço e na preensão, mostrou com um posicionamento de eletrodos ao redor do antebraço, que é possível minimizar os ruídos advindos dos outros músculos, quando analisados separadamente.

Através destes estudos, percebe-se que não há uma metodologia para o posicionamento dos eletrodos no antebraço, como pode ser verificado no estudo de Oskouei; Paulin; Carman (2013). 


\section{Objetivo}

O presente estudo teve por objetivo verificar a confiabilidade do posicionamento dos eletrodos nos músculos do antebraço na tarefa de preensão palmar entre o método proposto por Mogk e Keir e por Cram e Durie, por meio do teste e re-teste. 
METODOLOGIA 


\section{Metodologia}

O presente estudo teve caráter observacional e transversal de mensuração clínica de confiabilidade teste-reteste, tendo sido aprovado pelo comitê de ética em pesquisa do Hospital das Clinicas de Ribeirão Preto sob o número 13049/2013 ( Anexo A).

\subsection{Participantes}

Foram recrutados neste estudo 70 voluntários com uma amostra de conveniência, dos quais 30 preencheram os critérios de inclusão e exclusão divididos em grupo de pacientes e saudáveis. Como critérios de inclusão para o grupo de pacientes teriam que apresentar força de preensão mínima de $4 \mathrm{Kg} / \mathrm{F}$ avaliadas em um dinamômetro analógico da marca Jamar $^{\circledR}$ e amplitude de movimento para os dedos da mão dominante, que permitisse a realização da preensão palmar com lesão prévia tratada conservadoramente ou cirurgicamente na região do antebraço, punho ou da mão em tratamento no Centro de Reabilitação do Hospital das Clínicas de Ribeirão Preto da FMRP-USP, com liberação médica para o uso ativo do membro superior, sendo 13 homens e 2 mulheres com média de idade de 34,6 anos, sendo 14 destros e 1 canhoto. Foram excluídos os voluntários que se recusaram a serem submetidos aos procedimentos preparatórios para a SEMG como a tricotomia, a esfoliação da pele ou se os voluntários declarassem que não poderiam retornar para o re-teste. O grupo dos voluntários saudáveis não apresentavam nenhum trauma ou disfunção prévia no membro superior dominante, sendo compreendido por 13 mulheres e 2 homens com média de idade de 23,4 anos, 13 destros e 2 canhotos.

Todos os voluntários receberam informações sobre $\mathrm{O}$ experimento $\mathrm{e}$ assinaram o Termo de Consentimento Livre e Esclarecido (Apêndice A). 


\subsection{Posicionamento corporal durante os testes}

Os voluntários foram posicionados sentados em uma cadeira, com os joelhos e quadris posicionados à $90^{\circ}$ de flexão, com o membro superior dominante posicionado à $0^{\circ}$ de abdução e rotação de ombro, $90^{\circ}$ de flexão de cotovelo, antebraço posicionado em neutro, punho livre sem apoio. A tarefa realizada foi a preensão palmar com auxílio do dinamômetro analógico da marca Jamar ${ }^{\circledR}$ (Figura 1).

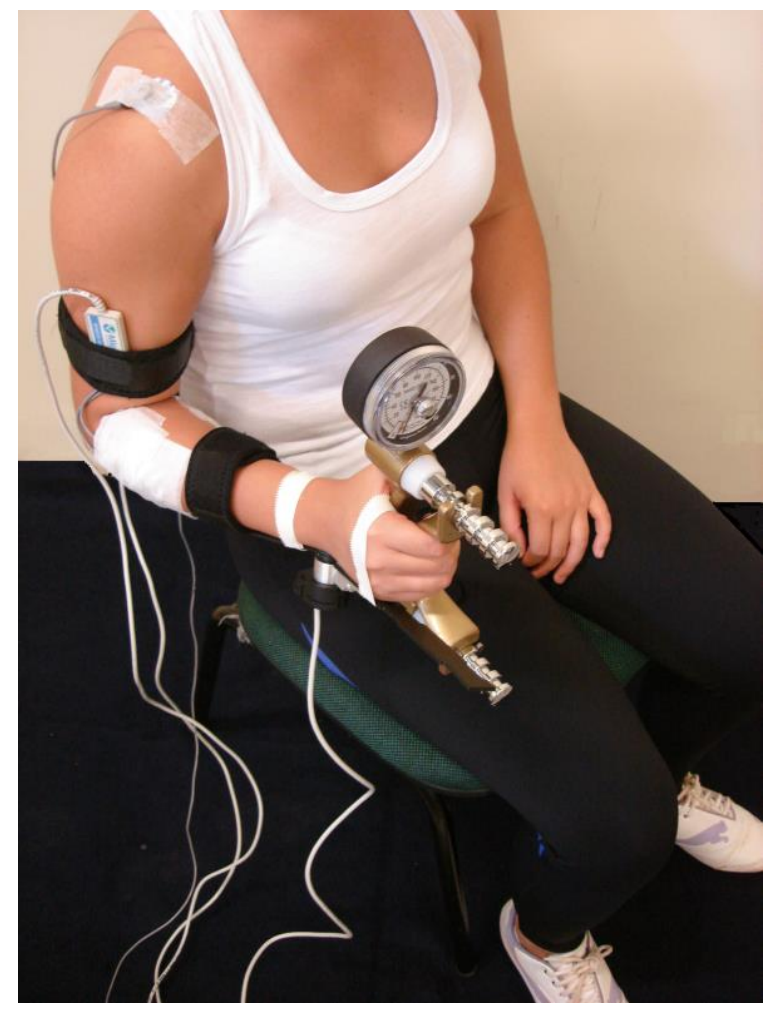

Figura 1: Posicionamento do sujeito e material utilizado durante a tarefa

\subsection{Posicionamento dos eletrodos}

Os eletrodos foram posicionados na região dos músculos extensor ulnar do carpo (EUC) (canal 01), extensor radial longo e curto do carpo (ERC) (canal 06), extensor comum dos dedos (ECD) (canal 07), flexor ulnar do carpo (FUC) (canal 05), flexor radial do carpo (FRC) (canal 02), flexor superficial dos dedos (FSD) (canal 4) e flexor profundo dos dedos (FPD) (canal 03). O preparo da pele ocorreu de acordo 
com as recomendações da SENIAM (HERMENS et. al., 2000) a pele foi tricotomizada, limpa com álcool 70\% e esfoliada para retirada de células mortas.

Utilizou-se duas técnicas de posicionamento dos eletrodos. A primeira conforme Mogk; Keir, (2003) propuzeram, que consiste em localizar por meio da palpação o músculo flexor radial do carpo, e seguir com 7 pares de eletrodos envolvendo o terço proximal do antebraço, apresentando uma distância de $3 \mathrm{~cm}$ de centro a centro dos eletrodos ( Figura 2)..

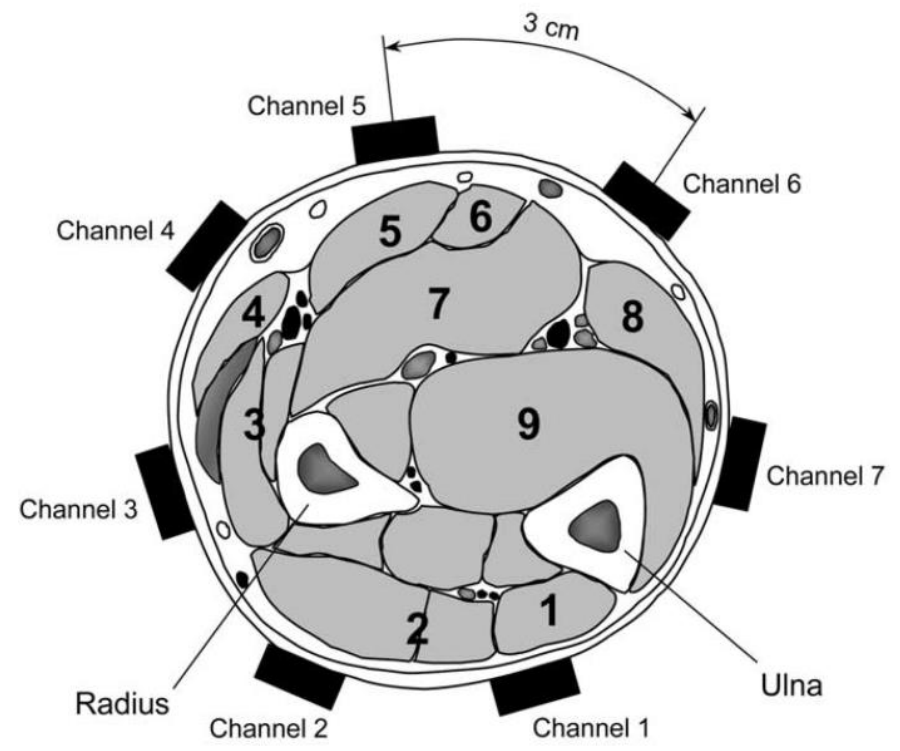

Figura 02 - Disposição dos eletrodos segundo método de Mogk e Keir (ano 2003): canal 1: extensor ulnar do carpo; canal 2: flexor ulnar do carpo; canal 3: flexor profundo dos dedos; canal 4: flexor superficial dos dedos; canal 5: flexor radial do carpo; canal 6: extensor radial do carpo; canal 7: extensor comum dos dedos.

A segunda ocorreu por meio da palpação do ventre muscular, segundo Cram; Durie, (2011), de acordo com esses autores a palpação foi realizada durante o movimento específico de cada músculo.

Os testes de força muscular seguiram os padrões propostos por Kendal et. al. (2005). Como pode ser visualizado na tabela a seguir. 


\begin{tabular}{|c|c|c|c|}
\hline Músculo & Fixação & Teste & Pressão \\
\hline $\begin{array}{l}\text { Extensor Comum } \\
\text { dos Dedos }\end{array}$ & $\begin{array}{l}\text { O examinador estabiliza o punho, } \\
\text { evitando a extensão. }\end{array}$ & $\begin{array}{c}\text { Estender as articulações } \\
\text { metacarpofalangianas do } 2^{\circ} \text { ao } 5^{\circ} \text { dedo } \\
\text { com as interfalangeanas relaxadas }\end{array}$ & $\begin{array}{l}\text { Contra as faces posteriores das falanges } \\
\text { proximais do } 2^{\circ} \text { ao } 5^{\circ} \text { dedo }\end{array}$ \\
\hline $\begin{array}{l}\text { Flexor Superficial } \\
\text { dos Dedos }\end{array}$ & $\begin{array}{l}\text { O examinador estabiliza a } \\
\text { articulação metacarpofalangeana, } \\
\text { com o punho em posição neutra }\end{array}$ & $\begin{array}{c}\text { Fletir a articulação interfalangeana } \\
\text { proximal com a interfalangeana distal } \\
\text { em extensão }\end{array}$ & Contra a face anterior da falange média \\
\hline $\begin{array}{l}\text { Flexor Profundo } \\
\text { dos Dedos }\end{array}$ & $\begin{array}{l}\text { Com o punho levemente em } \\
\text { extensão o examinador estabiliza } \\
\text { as falanges média e proximal }\end{array}$ & $\begin{array}{l}\text { Fletir a articulação interfalangeana } \\
\text { distal }\end{array}$ & $\begin{array}{l}\text { Contra a face anterior da falange distal em } \\
\text { direção da extensão }\end{array}$ \\
\hline $\begin{array}{l}\text { Flexor Radial do } \\
\text { Carpo }\end{array}$ & O antebraço é supinado e apoiado & Fletir o punho com desvio radial & $\begin{array}{c}\text { Contra a eminencia tenar em direção a } \\
\text { extensão e para o lado ulnar }\end{array}$ \\
\hline $\begin{array}{l}\text { Flexor Ulnar do } \\
\text { Carpo }\end{array}$ & $\begin{array}{l}\text { O antebraço tem que estar } \\
\text { totalmente supinado e apoiado }\end{array}$ & Fletir o punho com desvio ulnar & $\begin{array}{c}\text { Contra a eminencia hipotênar em direção a } \\
\text { extensão e para o lado radial }\end{array}$ \\
\hline $\begin{array}{l}\text { Extensor Radial } \\
\text { Longo e Curto do } \\
\text { Carpo }\end{array}$ & O antebraço é pronado e apoiado & Estender o punho com desvio radial & $\begin{array}{l}\text { Contra a face posterior da mão, entre o } 2^{\circ} \text { e o } \\
\text { 3o metacarpo, em direção a flexão e para o } \\
\text { lado ulnar }\end{array}$ \\
\hline $\begin{array}{l}\text { Extensor Ulnar } \\
\text { do Carpo }\end{array}$ & O antebraço é pronado e apoioado & Estender o punho com desvio ulnar & $\begin{array}{l}\text { Contra a face posterior da mão, ao longo do 50 } \\
\text { metacarpo, em direção a flexão para o lado } \\
\text { radial }\end{array}$ \\
\hline
\end{tabular}

Tabela 01: Teste de força muscular segundo Kendall et. al. (2005) 
A aquisição do sinal EMG ocorreu por meio da realização da tarefa de preensão palmar com o dinamômetro analógico da marca Jamar ${ }^{\circledR}$, sendo realizado três aquisições com contrações máxima voluntária adquirida no tempo de 10 segundos cada e tempo de 3 minutos de descanso entre as tarefas, que foram realizadas sob comando verbal do avaliador.

\subsection{Normalização do sinal}

Previamente à coleta, os dados foram normalizados por meio de 1 contração voluntária máxima de 6 segundos, com período de repouso de 1 minuto, no qual foi realizada a tarefa de preensão palmar no dispositivo dinamômetro analógico da marca Jamar®. (BURDEN; BARLETT, 1999; KONG et. al., 2010).

\subsection{Procedimento de registro eletromiográfico}

O estudo teve o formato de teste/re-teste. A coleta foi dividida em 2 dias, sendo no primeiro dia realizado uma coleta formalizando o teste e no segundo dia após 24 horas - ocorreu a segunda coleta formalizando o re-teste e assim o interavaliador. A análise foi feita pelo root means square (RMS). O eletromiógrafo utilizado foi o Miotool 400 da marca Miotec ${ }^{\circledR}$, com eletrodos passivos bipolares autoadesivos com distância de $20 \mathrm{~mm}$ entre os centros. O software utilizado foi o Miograph 2.0 da empresa Miotec $^{\circledR}$.

\subsection{Análise Estatística}

A confiabilidade teste-reteste das medidas para ambos os métodos foi realizada usando o programa SPSS $®$ (versão 20 ) para Windows ${ }^{\circledR}$. Foram calculados - ICC e o SEM (Standard Error of Measurement) pelas médias e desvio padrão das três medidas do valor do RMS normalizado, com intervalo de confiança de $95 \%$. Os valores do ICC foram considerados pobres entre $0-0,49$, moderados entre $0,5-0,69$, altos entre 0,70-0,89 e excelentes acima de 0,9 Adicionalmente, foi utilizado o 
método de Bland-Altman visando determinar erros sistemáticos entre os valores médios e a diferença entre os valores entre os métodos. Este modelo consiste em analisar por meio de um gráfico de dispersão a concordância entre duas variáveis ${ }^{2,16,17,18}$ calculados pelo programa MedCalc®, versão 14. 
RESULTADOS 


\section{Resultados}

\subsection{Valores de Amplitude Eletromiográfica - RMS}

A média e o desvio padrão dos valores de RMS normalizados ( $\mathrm{UV}$ ) dos canais avaliados nos dois tipos de posicionamento, nos dois grupos - pacientes e não pacientes - no teste e re-teste, estão descritos nas tabelas abaixo.

\begin{tabular}{ccccc}
\hline Canais & \multicolumn{2}{c}{ Teste } & \multicolumn{2}{c}{ Re-Teste } \\
& Mogk & Cram & Mogk & Cram \\
\hline EUC & $0,38(0,16)$ & $0,32(0,11)$ & $0,36(0,10)$ & $0,32(0,08)$ \\
FUC & $0,34(0,09)$ & $0,32(0,09)$ & $0,33(0,02)$ & $0,33(0,01)$ \\
FPD & $0,34(0,08)$ & $0,35(0,09)$ & $0,34(0,00)$ & $0,33(0,00)$ \\
FSD & $0,35(0,11)$ & $0,35(0,11)$ & $0,36(0,01)$ & $0,35(0,00)$ \\
FRC & $0,36(0,13)$ & $0,39(0,14)$ & $0,39(0,00)$ & $0,45(0,00)$ \\
ERC & $0,34(0,10)$ & $0,34(0,13)$ & $0,33(0,00)$ & $0,36(0,00)$ \\
ECD & $0,35(0,12)$ & $0,35(0,12)$ & $0,36(0,03)$ & $0,38(0,02)$ \\
\hline
\end{tabular}

Tabela 02. Média e Desvio Padrão dos valores de RMS normalizados (UV) dos grupos de pacientes e não pacientes, obtidos no teste e re-teste dos posicionamentos Mogk e Cram 


\begin{tabular}{ccccc}
\hline Canais & \multicolumn{2}{c}{ Teste } & \multicolumn{2}{c}{ Re-Teste } \\
\hline & Mogk & Cram & Mogk & Cram \\
\hline EUC & $0,42(0,19)$ & $0,35(0,11)$ & $0,40(0,12)$ & $0,35(0,08)$ \\
FUC & $0,38(0,09)$ & $0,34(0,10)$ & $0,38(0,07)$ & $0,35(0,10)$ \\
FPD & $0,34(0,07)$ & $0,35(0,06)$ & $0,34(0,07)$ & $0,33(0,05)$ \\
FSD & $0,35(0,12)$ & $0,34(0,11)$ & $0,38(0,10)$ & $0,37(0,07)$ \\
FRC & $0,39(0,14)$ & $0,42(0,13)$ & $0,41(0,19)$ & $0,50(0,19)$ \\
ERC & $0,37(0,10)$ & $0,33(0,10)$ & $0,32(0,13)$ & $0,35(0,08)$ \\
ECD & $0,37(0,13)$ & $0,32(0,06)$ & $0,35(0,07)$ & $0,37(0,09)$ \\
\hline
\end{tabular}

Tabela 03. Média e Desvio Padrão dos valores de RMS normalizados (uV) do grupo não pacientes, obtidos no teste e re-teste dos posicionamentos Mogk e Cram.

\begin{tabular}{ccccc}
\hline Canais & \multicolumn{2}{c}{ Teste } & \multicolumn{2}{c}{ Re-Teste } \\
\hline & Mogk & Cram & Mogk & Cram \\
\hline EUC & $0,34(0,13)$ & $0,29(0,10)$ & $0,32(0,07)$ & $0,29(0,06)$ \\
FUC & $0,30(0,07)$ & $0,30(0,08)$ & $0,28(0,05)$ & $0,30(0,06)$ \\
FPD & $0,33(0,08)$ & $0,36(0,11)$ & $0,33(0,07)$ & $0,33(0,08)$ \\
FSD & $0,36(0,11)$ & $0,36(0,10)$ & $0,33(0,10)$ & $0,33(0,08)$ \\
FRC & $0,33(0,11)$ & $0,35(0,14)$ & $0,36(0,13)$ & $0,40(0,20)$ \\
ERC & $0,32(0,11)$ & $0,35(0,16)$ & $0,33(0,17)$ & $0,36(0,15)$ \\
ECD & $0,33(0,12)$ & $0,37(0,16)$ & $0,37(0,12)$ & $0,38(0,14)$ \\
\hline
\end{tabular}

Tabela 04. Média e Desvio Padrão dos valores de RMS normalizados (uV) do grupo pacientes, obtidos no teste e re-teste dos posicionamentos Mogk e Cram. 
5.2. Valores de confiabilidade:

\begin{tabular}{lllll}
\hline Canais & \multicolumn{2}{c}{ Mogk } & \multicolumn{2}{c}{ Cram } \\
\hline & ICC & SEM & ICC & SEM \\
\hline EUC & 0,902 & 0,04 & 0,902 & 0,03 \\
FUC & 0,903 & 0,02 & 0,958 & 0,01 \\
FPD & 0,918 & 0,02 & 0,840 & 0,03 \\
FSD & 0,785 & 0,05 & 0,782 & 0,04 \\
FRC & 0,870 & 0,05 & 0,844 & 0,07 \\
ERC & 0,783 & 0,06 & 0,862 & 0,04 \\
ECD & 0,890 & 0,03 & 0,797 & 0,05 \\
\hline
\end{tabular}

Tabela 05. Valores do coeficiente de correlação intraclasse (ICC) para confiabilidade inter-dia e erro padrão de medida (SEM) referente aos valores de RMS normalizados de ambos os grupos.

Verifica-se na tabela 05 que tanto o posicionamento realizado por Mogk e por Cram obtiveram excelentes resultados na confiabilidade, apresentando erro padrão de medida baixos. 


\begin{tabular}{lllll}
\hline Canais & \multicolumn{2}{c}{ Mogk } & \multicolumn{2}{c}{ Cram } \\
\hline & ICC & SEM & ICC & SEM \\
\hline EUC & 0,711 & 0,05 & 0,833 & 0,03 \\
FUC & 0,674 & 0,03 & 0,918 & 0,02 \\
FPD & 0,931 & 0,02 & 0,822 & 0,04 \\
FSD & 0,594 & 0,06 & 0,821 & 0,03 \\
FRC & 0,728 & 0,06 & 0,554 & 0,11 \\
ERC & 0,699 & 0,07 & 0,862 & 0,05 \\
ECD & 0,880 & 0,04 & 0,768 & 0,07 \\
\hline
\end{tabular}

Tabela 06. Valores do coeficiente de correlação intraclasse (ICC) para confiabilidade inter-dia e erro padrão de medida (SEM) referente aos valores de RMS normalizados do grupo pacientes.

Quando analisado somente o grupo de pacientes, como segue na tabela 06, percebe-se que somente duas medidas obtiveram excelente índice, como é o caso dos FPD e ECD. Enquanto os outros canais obtiveram boa correlação no posicionamento proposto por Mogk.

No posicionamento proposto por Cram ocorre que somente no FUC apresentou boa correlação, enquanto os outros canais apresentaram excelente correlação. 


\begin{tabular}{lllll}
\hline Canais & \multicolumn{2}{c}{ Mogk } & \multicolumn{2}{c}{ Cram } \\
\hline & ICC & SEM & ICC & SEM \\
\hline EUC & 0,933 & 0,042 & 0,919 & 0,027 \\
FUC & 0,907 & 0,027 & 0,972 & 0,017 \\
FPD & 0,905 & 0,025 & 0,890 & 0,022 \\
FSD & 0,878 & 0,043 & 0,758 & 0,052 \\
FRC & 0,913 & 0,049 & 0,950 & 0,039 \\
ERC & 0,870 & 0,040 & 0,875 & 0,035 \\
ECD & 0,911 & 0,033 & 0,864 & 0,031 \\
\hline
\end{tabular}

Tabela 07. Valores do coeficiente de correlação intraclasse (ICC) para confiabilidade intra-dia e erro padrão de medida (SEM) referente aos valores de RMS normalizados do grupo não pacientes.

Quando se analisa o grupo de não pacientes verifica uma excelente confiabilidade no posicionamento proposto por Mogk e no posicionamento proposto por Cram.

\subsection{Gráfico de Dispersão de Bland-Altman}

O método de Bland-Altman consiste em analisar por meio de um gráfico de dispersão avaliar a concordância entre duas variáveis, na qual analisa a diferença das duas variáveis e a média das duas (BLAND; ALTMAN, 1999).

$\mathrm{Na}$ figura 03, verifica-se um viés muito baixo, isto é, apresenta somente dois outliers e também apresenta concordância e correlação entre as medidas, já que em sua maioria está presente entre os valores de desvio padrão. 


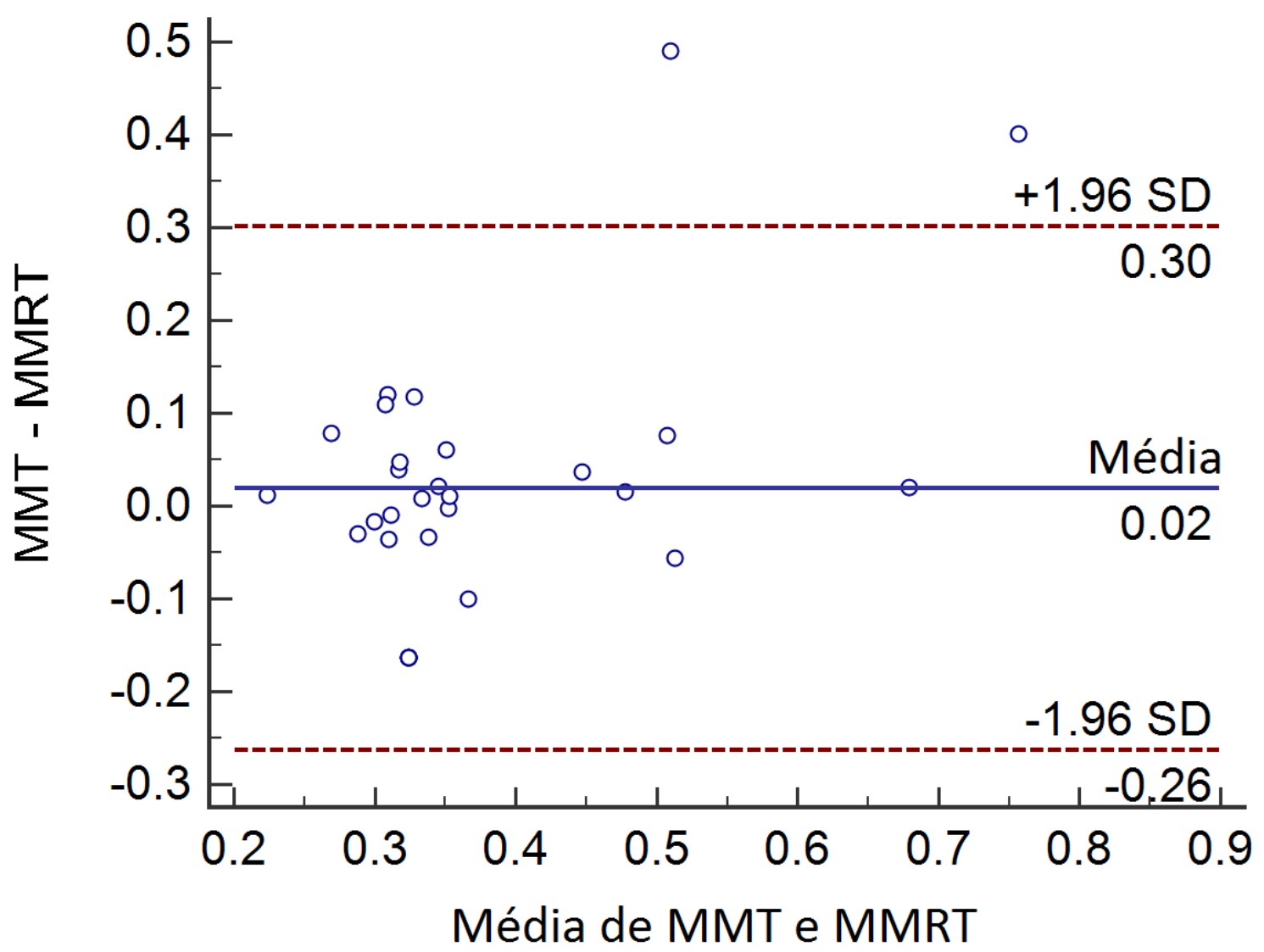

Figura 03: Gráfico de dispersão de concordância entre medidas de BlandAltman no método Mogk do EUC na captação eletromiográfica nos modos teste e reteste em ambos os grupos. MMT - método Mogk teste; MMRT - método Mogk Reteste.

$\mathrm{Na}$ análise do FRC nota-se que também há uma correlação e uma concordância nos valores, porém as medidas se apresentam mais dispersas, mostrando que a correlação é menor que o EUC. O viés é também irrelevante, revelando novamente uma ótima concordância. 


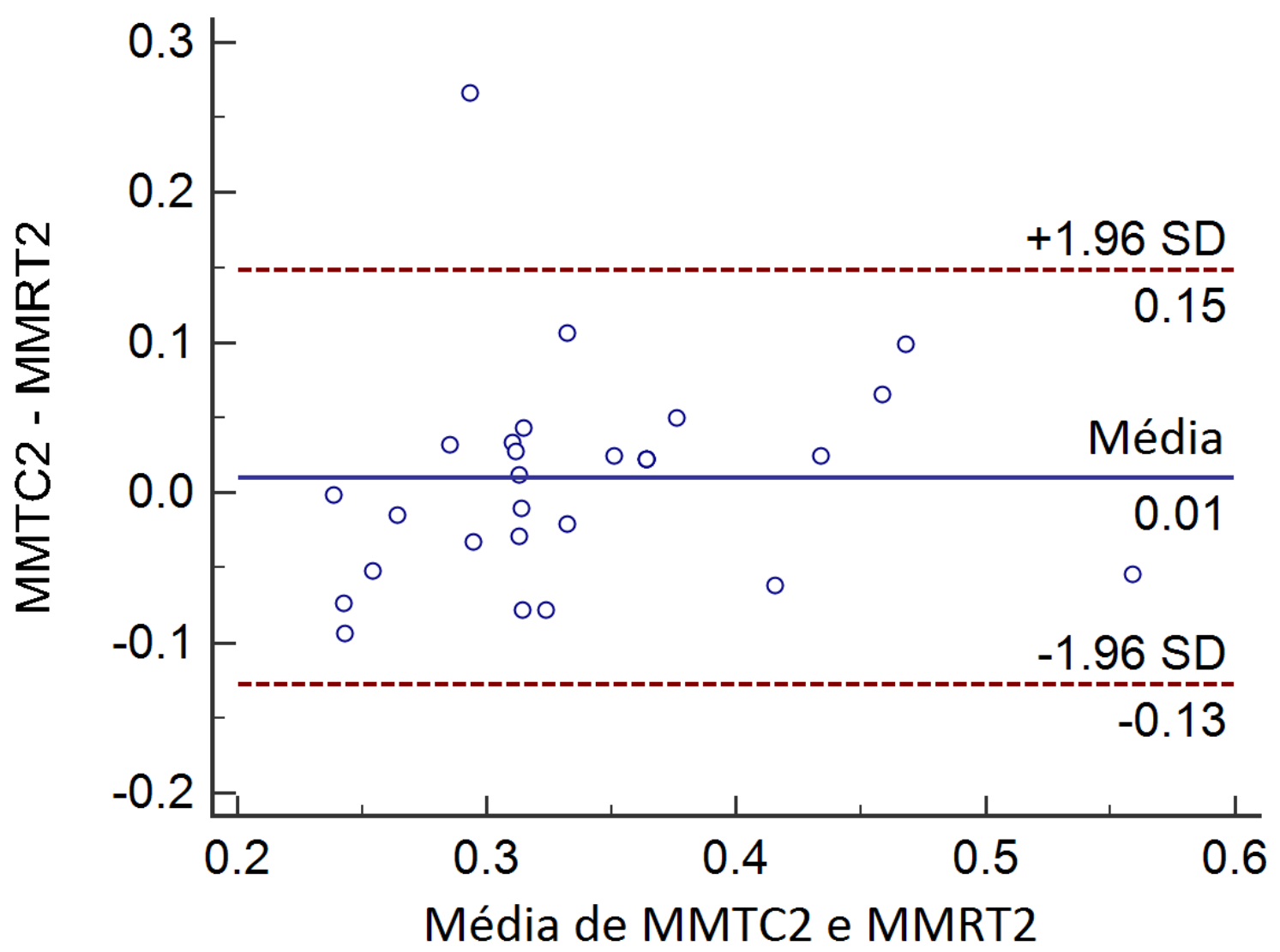

Figura 4: Gráfico de dispersão de concordância entre medidas de BlandAltman no método Mogk do FUC na captação eletromiográfica nos modos teste e reteste em ambos os grupos. MMTC - método Mogk teste canal 2; MMRT 2 - método Mogk Re-teste canal 2.

$\mathrm{Na}$ figura 05 revela um viés ausente, isto é sem outliers, mostrando uma ótima concordância entre os valores, porém se verifica uma baixa correlação, pois os valores, mesmo estando dentro do intervalo de confiança, se apresentam dispersos. 


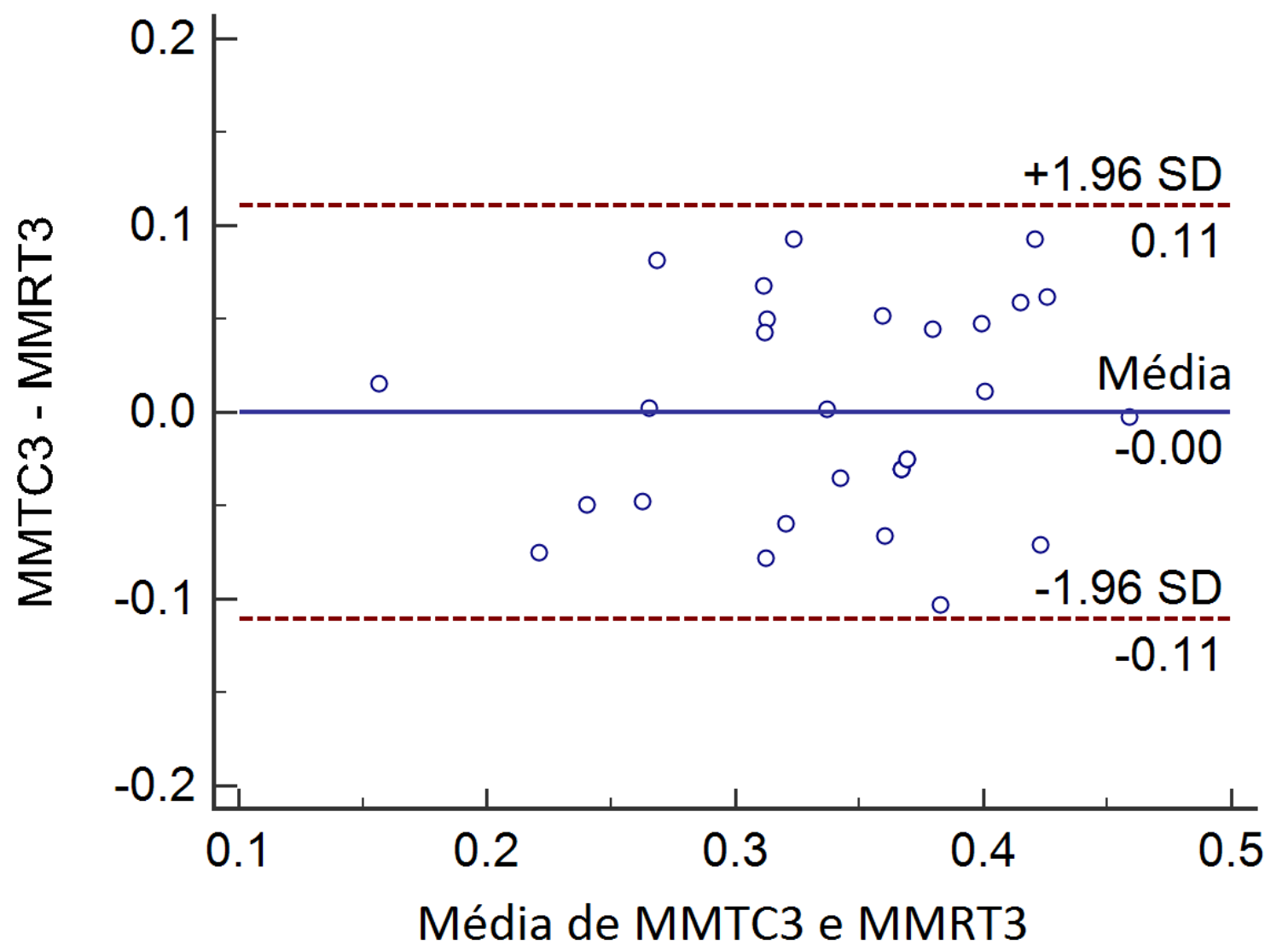

Figura 05: Gráfico de dispersão de concordância entre medidas de BlandAltman no método Mogk do FPD na captação eletromiográfica nos modos teste e reteste ambos os grupos. MMTC3 - método Mogk teste canal 3; MMRT3 - método Mogk Re-teste canal 3.

Na Figura 06 novamente percebe uma ótima concordância entre as medidas, já que o viés é nulo. Há uma grande correlação entre as medidas já que a maioria dos valores estão próximos da média. 


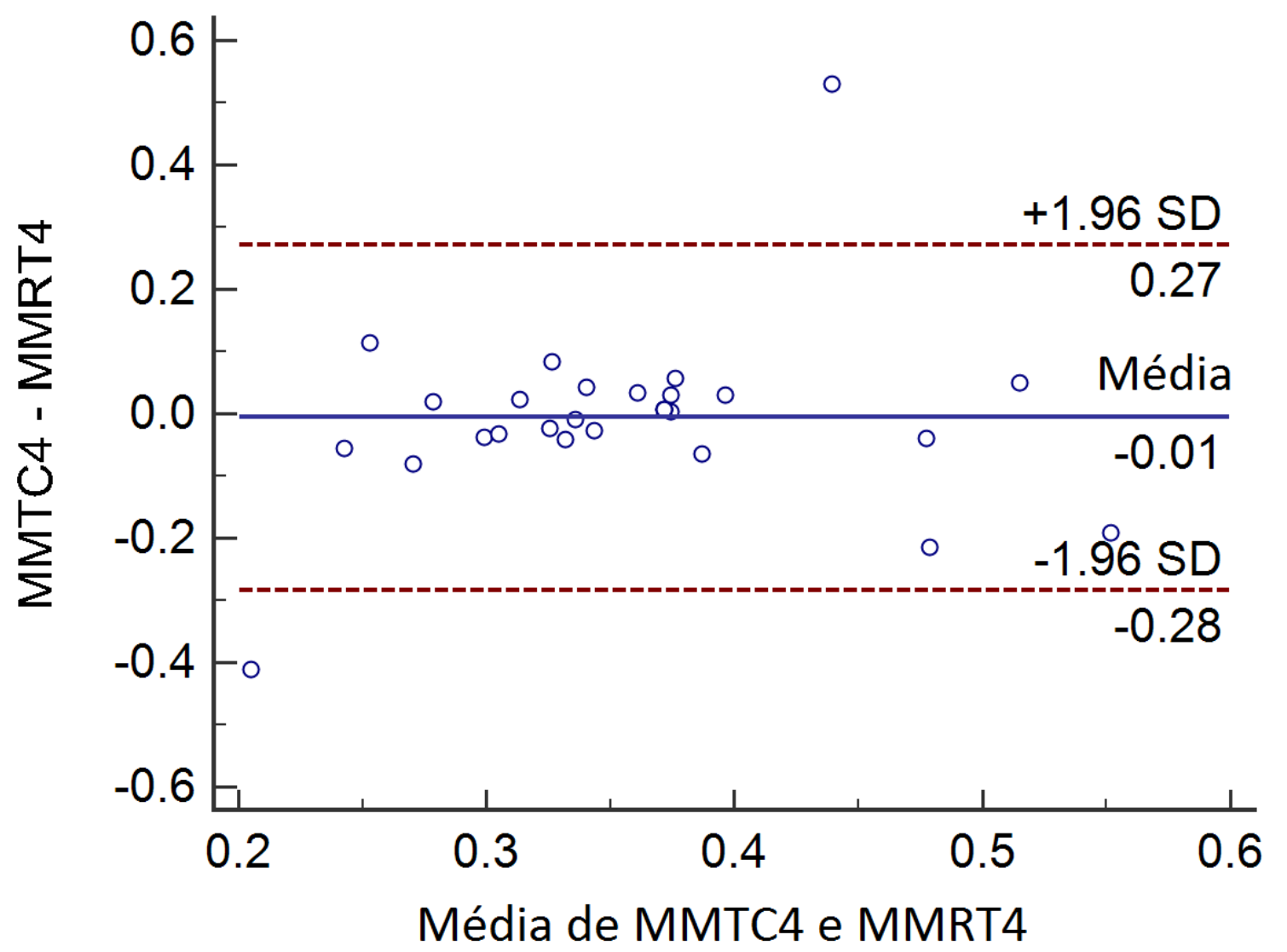

Figura 06: Gráfico de dispersão de concordância entre medidas de BlandAltman no método Mogk do FSD na captação eletromiográfica nos modos teste e reteste em ambos os grupos. MMTC4 - método Mogk teste canal 4; MMRT4 - método Mogk Re-teste canal 4.

O FUC apresenta concordância entre as medidas, mesmo apresentando um outlier, com isso o viés é muito baixo (Figura 07). 


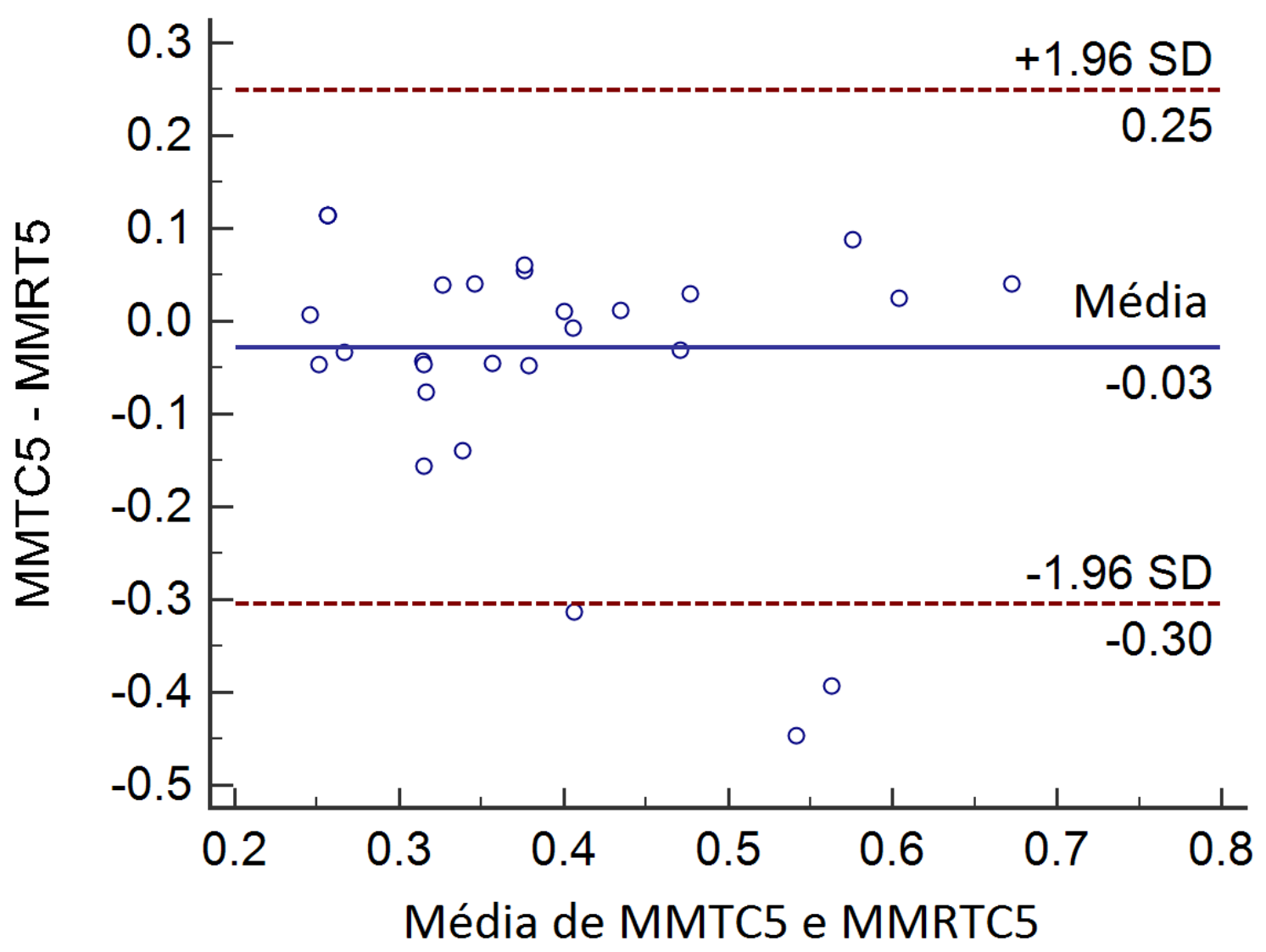

Figura 07:Gráfico de dispersão de concordância entre medidas de BlandAltman no método Mogk do FRC na captação eletromiográfica nos modos teste e reteste em ambos os grupos. MMTC5 - método Mogk teste canal 5; MMRT5 - método Mogk Re-teste canal 5.

$\mathrm{Na}$ figura 08, também apresenta correlação das medidas, porém neste caso 0 viés deixa a concordância mais baixa. 


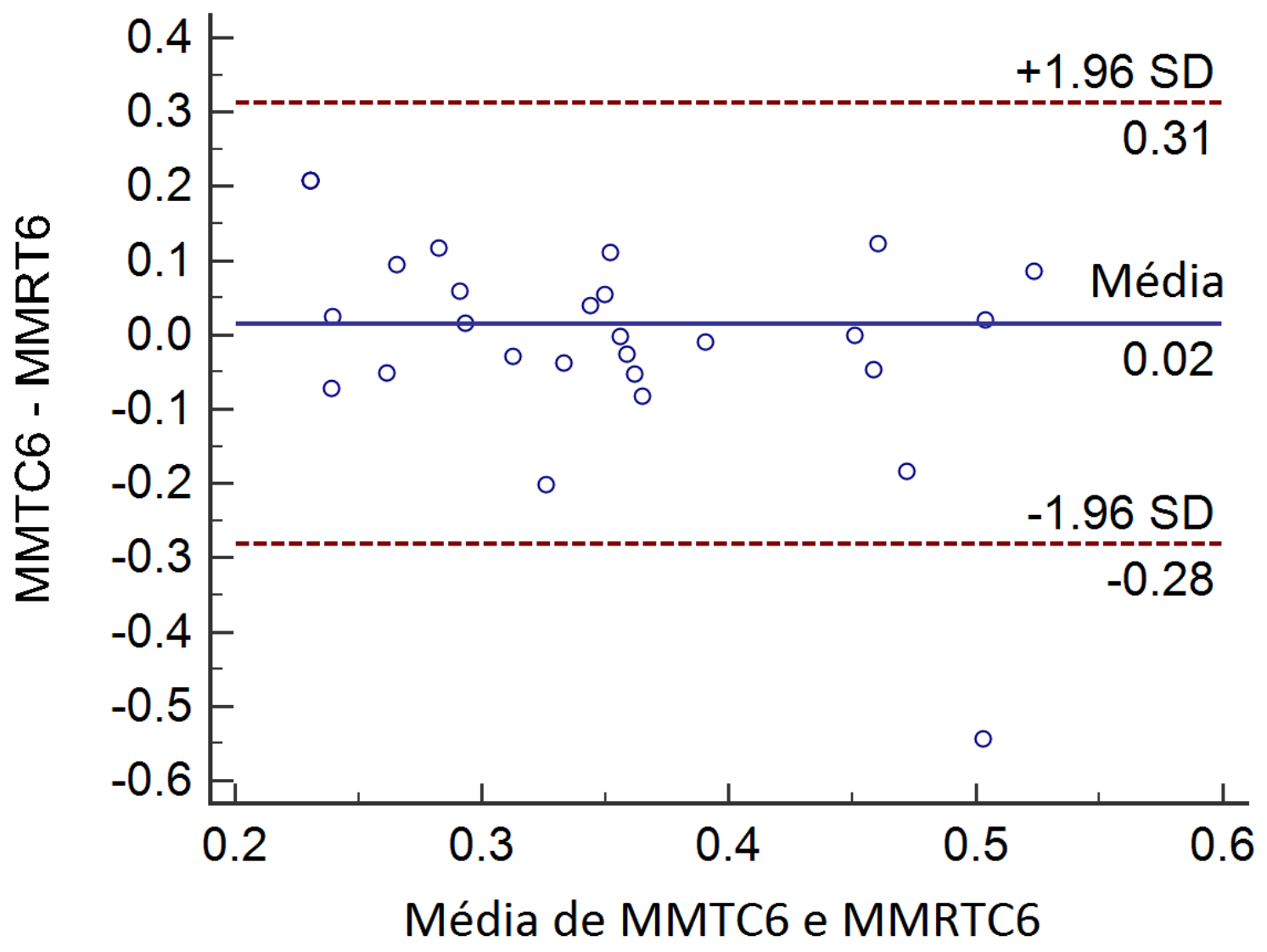

Figura 08: Gráfico de dispersão de concordância entre medidas de BlandAltman no método Mogk do ERC na captação eletromiográfica nos modos teste e reteste em ambos os grupos. MMTC6 - método Mogk teste canal 6; MMRT6 - método Mogk Re-teste canal 6.

O ECD, apresenta concordância e correlação dos valores, pois o viés é nulo e os valores se aproximam da média. 


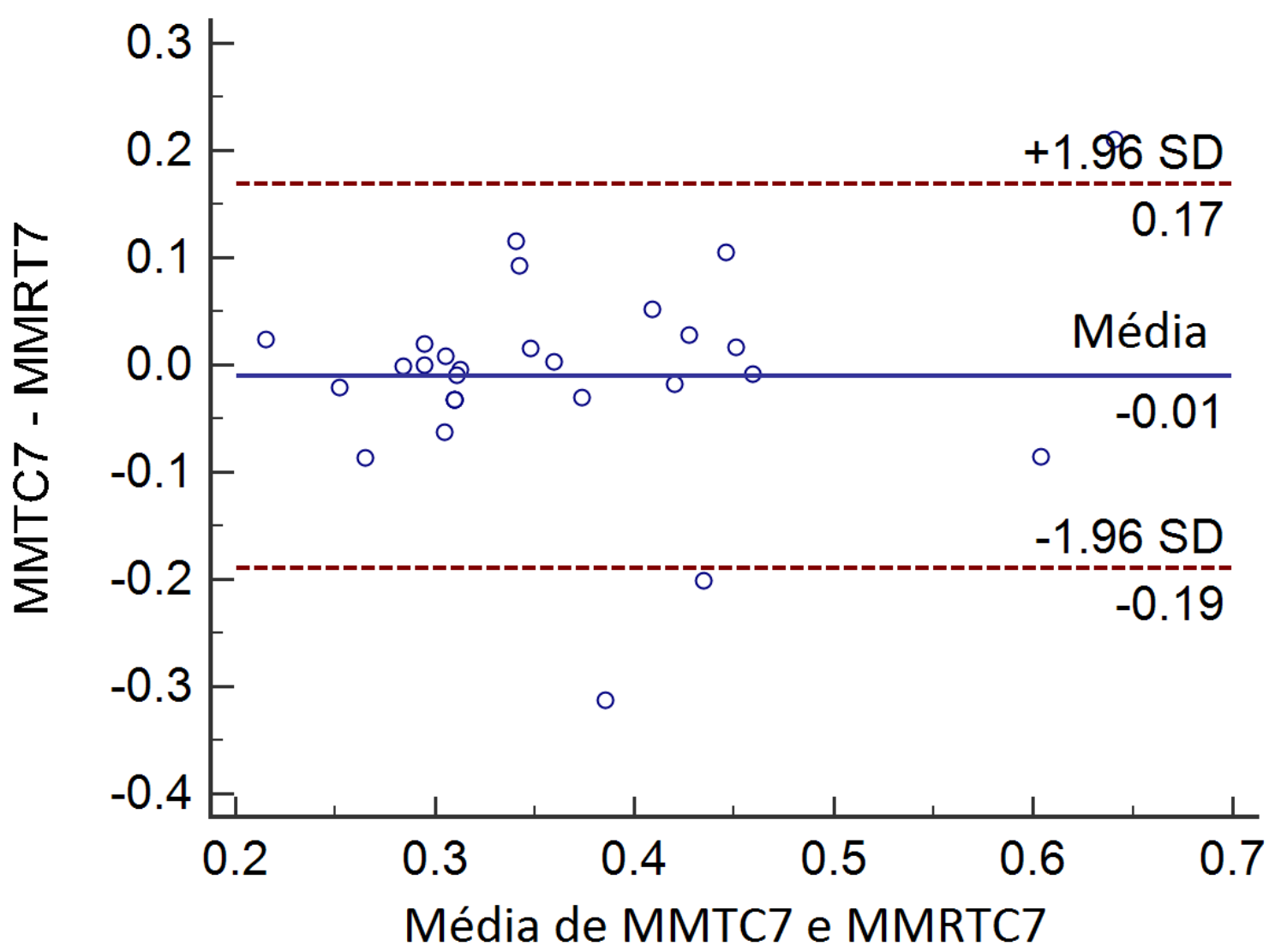

Figura 09: Gráfico de dispersão de concordância entre medidas de BlandAltman no método Mogk do ECD na captação eletromiográfica nos modos teste e reteste em ambos os grupos. MMTC7 - método Mogk teste canal 7; MMRT7 - método Mogk Re-teste canal 7.

A figura 10 mostra que há concordância mas a correlação é comprometida, pois os valores se apresentam dispersos, mesmo estando dentro do intervalo de confiança. 


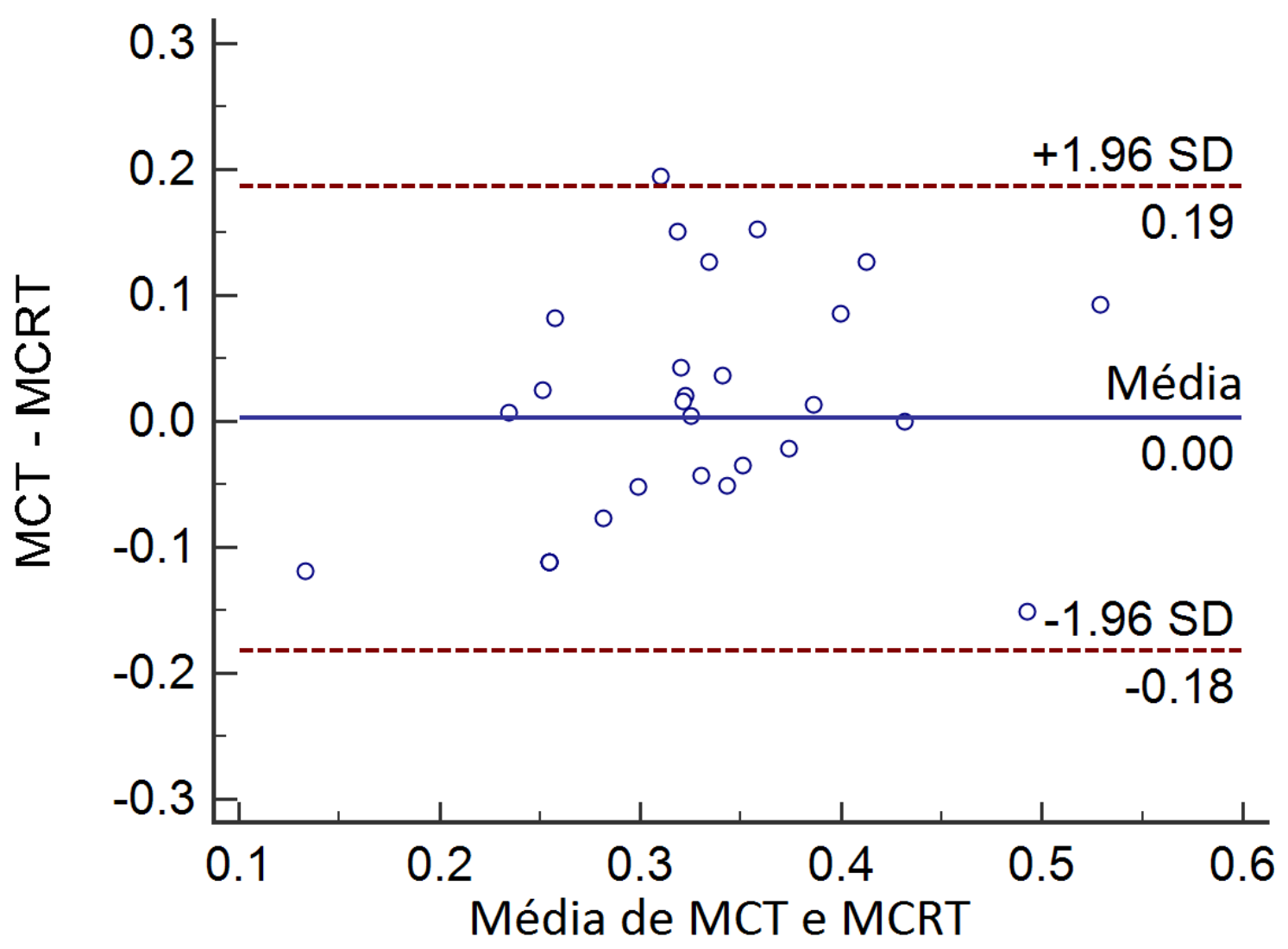

Figura 10: Gráfico de dispersão de concordância entre medidas de BlandAltman no método Cram do EUC na captação eletromiográfica nos modos teste e reteste em ambos os grupos. MCT - método Cram teste; MCRT - método Cram Reteste.

Como na figura 10 a figura 11 apresenta valores dentro do intervalo de confiança de $95 \%$ e o viés é quase nulo, apresentando um valor como outlier, mostrando concordância e correlação. 


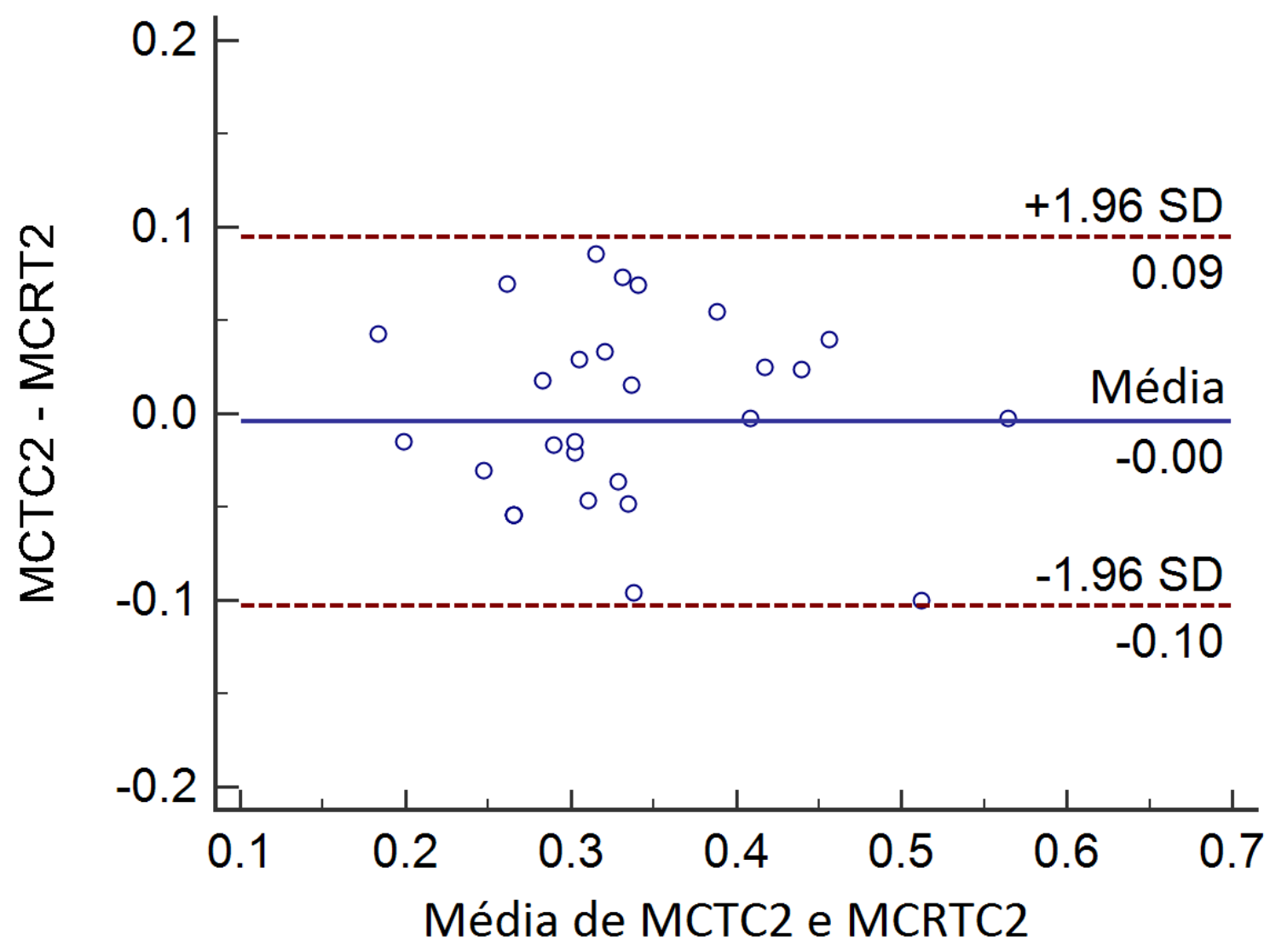

Figura 11: Gráfico de dispersão de concordância entre medidas de BlandAltman no método Cram do FUC na captação eletromiográfica nos modos teste e reteste em ambos os grupos. MCTC2 - método Cram teste canal 2; MCRTC 2 método Cram Re-teste canal 2.

No caso da figura 12, há também uma concordância e uma correlação entre as medidas, verificado pelos valores estarem bem próximos a média. 


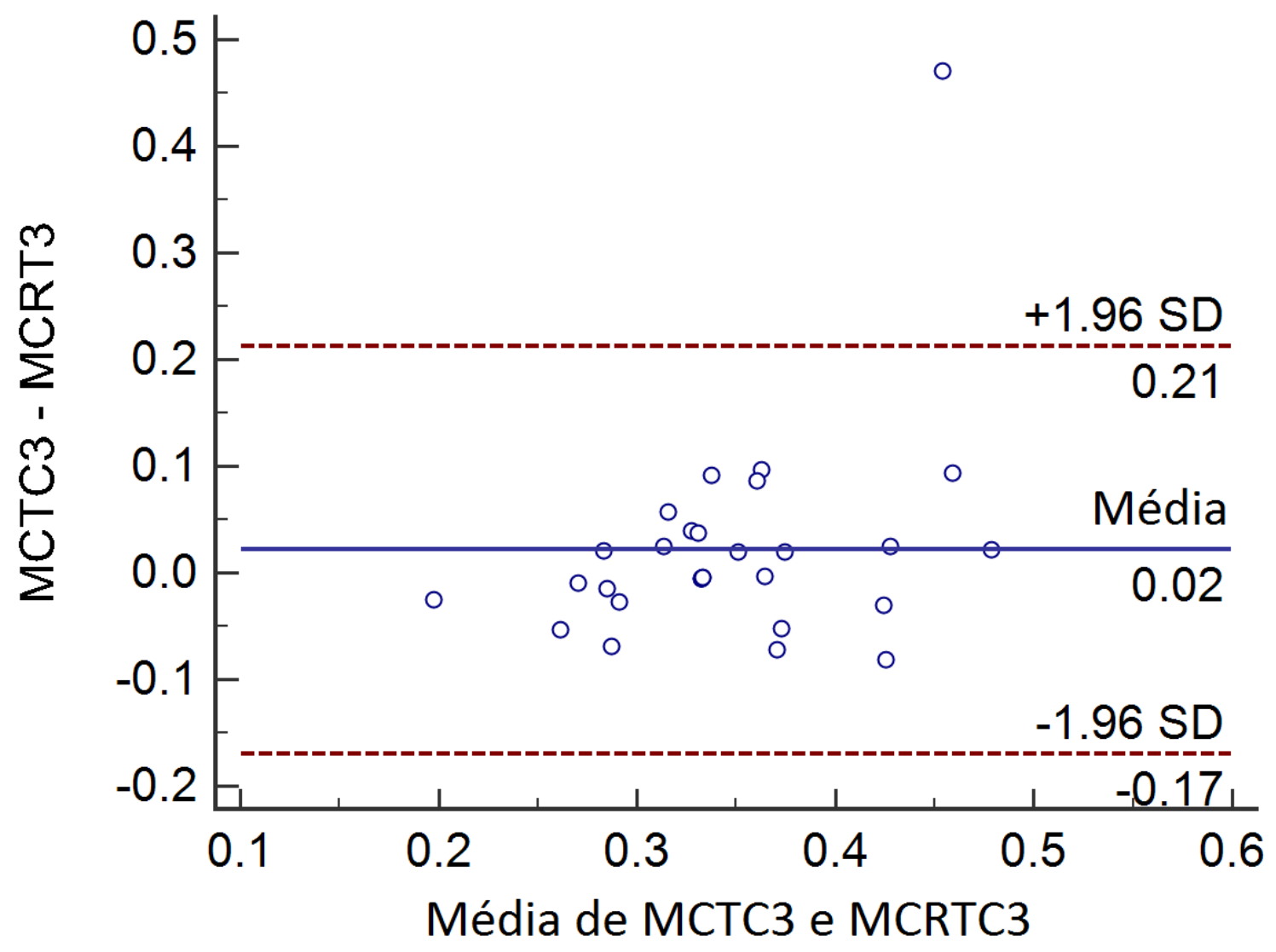

Figura 12: Gráfico de dispersão de concordância entre medidas de BlandAltman no método Cram do FPD na captação eletromiográfica nos modos teste e reteste em ambos os grupos. MCTC3 - método Cram teste canal 3; MCRTC3 método Cram Re-teste canal 3.

O FSD revela que há também uma concordância e uma correlação entre as medidas. 


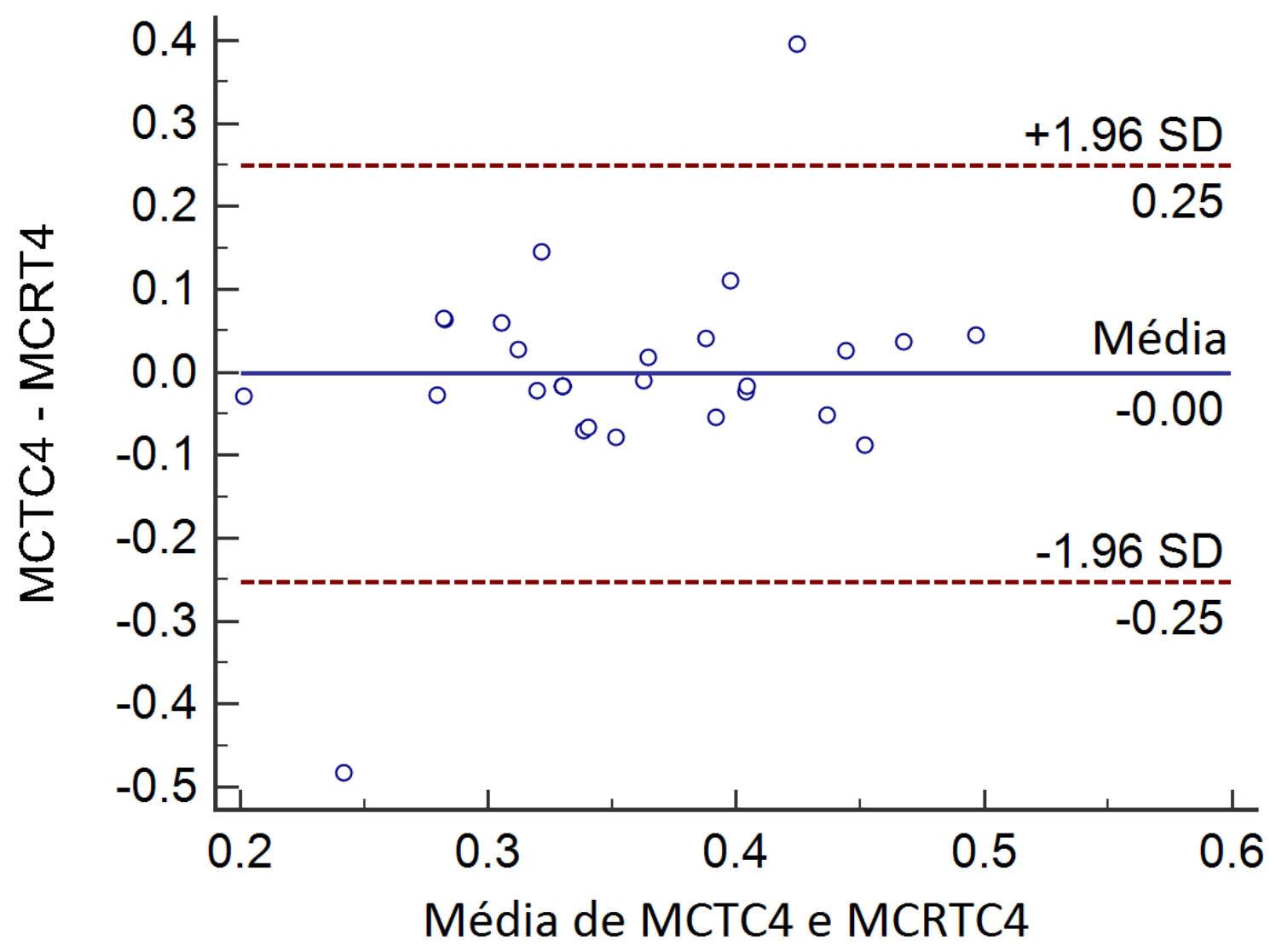

Figura 13: Gráfico de dispersão de concordância entre medidas de BlandAltman no método Cram do FSD na captação eletromiográfica nos modos teste e reteste em ambos os grupos. MCTC4 - método Cram teste canal 4; MCRTC4 método Cram Re-teste canal 4.

Na figura 14 revela que há uma concordância e correlação entre as medidas. 


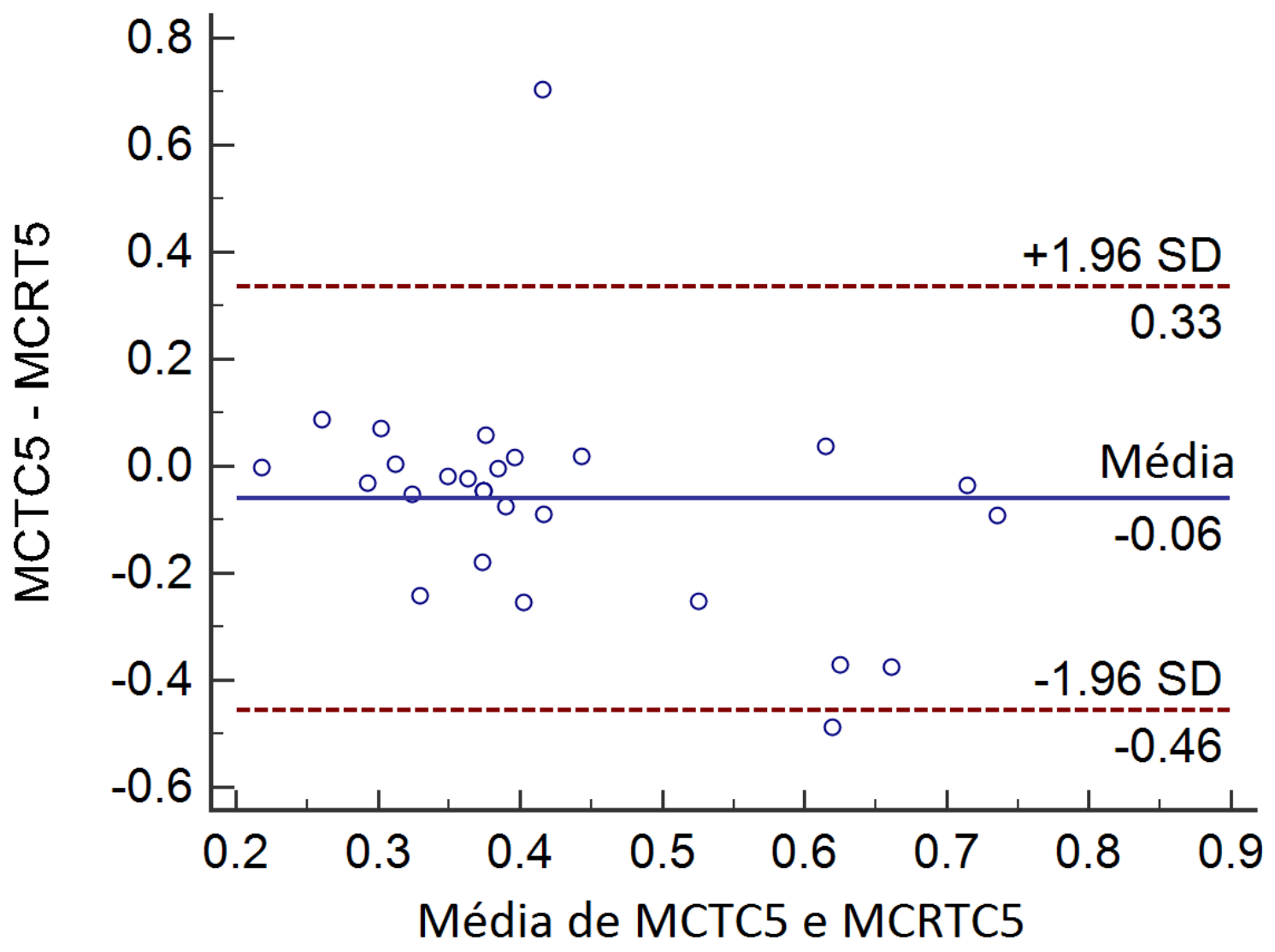

Figura 14: Gráfico de dispersão de concordância entre medidas de BlandAltman no método Cram do FRC na captação eletromiográfica nos modos teste e reteste em ambos os grupos. MCTC5 - método Cram teste canal 5; MCRTC5 método Cram Re-teste canal 5.

A figura 15 revela que houve uma concordância entre os valores, porém, novamente, há uma correlação moderada, já que os valores se apresentam dispersos pelo intervalo de confiança. 


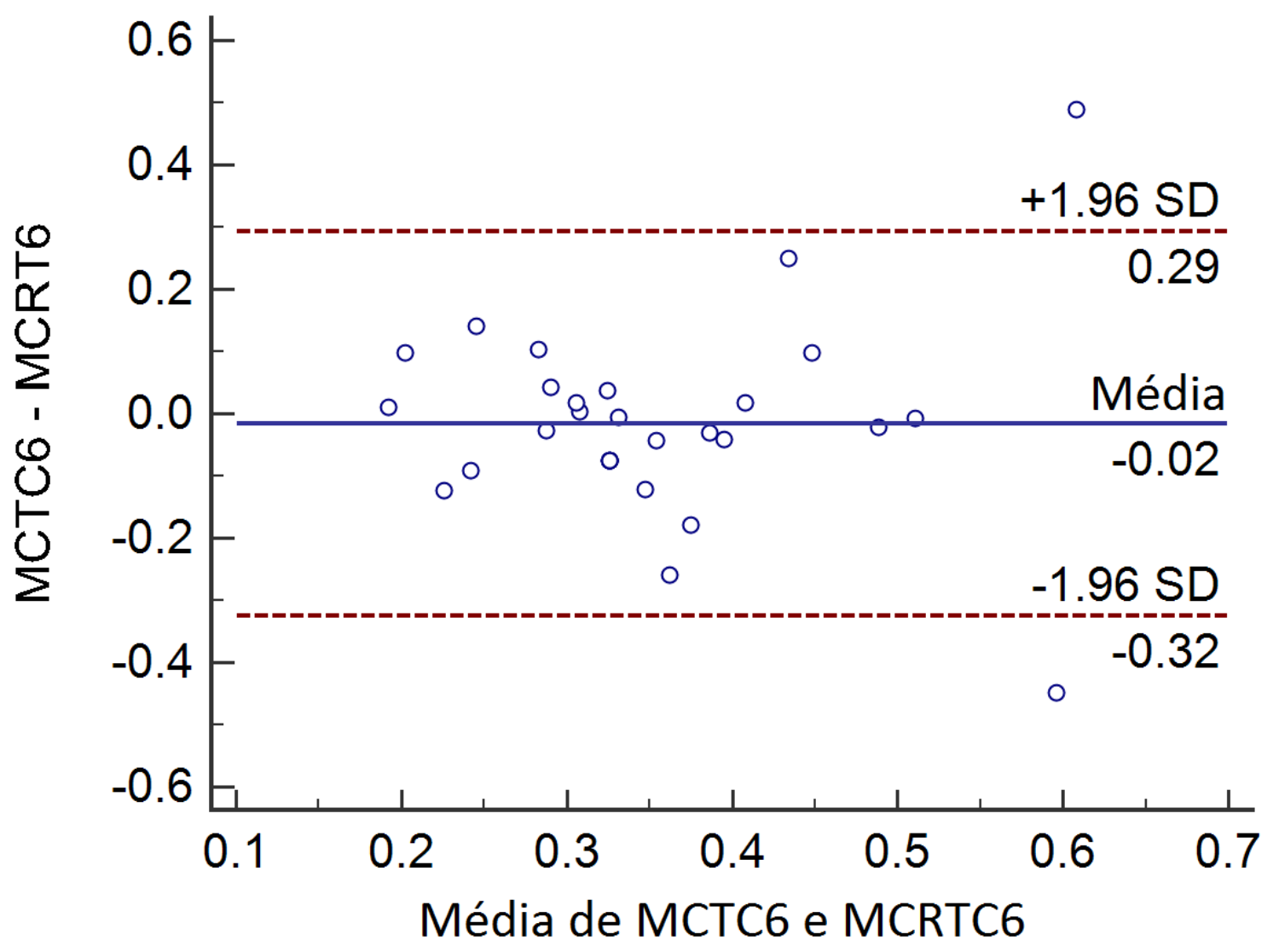

Figura 15: Gráfico de dispersão de concordância entre medidas de BlandAltman no método Cram do ERC na captação eletromiográfica nos modos teste e reteste em ambos os grupos. MCTC6 - método Cram teste canal 6; MCRTC6 método Cram Re-teste canal 6.

Neste caso os valores estão muito dispersos, revelando pouca correlação, porém houve concordância entre os valores. 


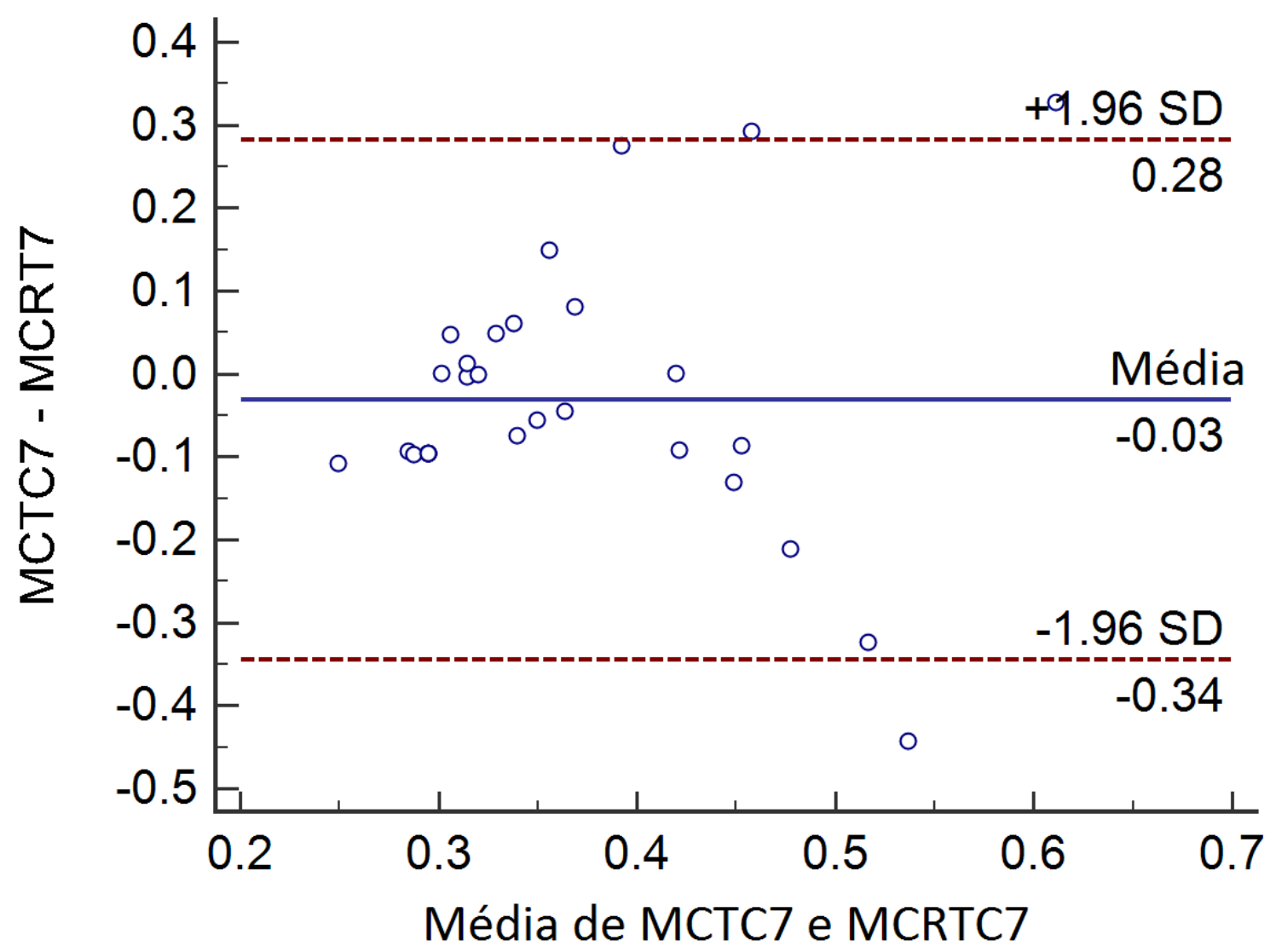

Figura 16: Gráfico de dispersão de concordância entre medidas de BlandAltman no método Cram do ECD na captação eletromiográfica nos modos teste e reteste em ambos os grupos. MCTC7 - método Cram teste canal 7; MCRTC7 método Cram Re-teste canal 7.

Analisando todos os gráficos, podemos inferir que em todos os casos houve concordância entre os valores, porém no método de posicionamento proposto por Mogk, há 2 canais em que a correlação está prejudicada, devido aos seus valores estarem dispersos.

Como ocorre no método proposto por Cram; Durie (2011), em que todos os gráficos revelaram concordância, mas em 3 deles a grande dispersão dos valores, revela uma correlação mais baixa. 


\section{Discussão}

O objetivo do presente estudo foi de analisar a confiabilidade teste re-teste do posicionamento dos eletrodos de superfície de eletromiografia, sendo utilizado os métodos de Cram; Durie (2011) e de Mogk; Keir (2003).

Diversos estudos atribuem a alta inconsistência da medida ao fato de apresentarem uma imprecisão no reposicionamento dos eletrodos. (ZAKARIA; KRAMER; HARBURN, 1996; NG et. al., 2003).

Tal fato está intimamente relacionado com os estudos de confiabilidade, na qual é a melhor maneira de se verificar, se a ferramenta ou o método, é reprodutível, e assim poder ser utilizado por diferentes avaliadores sem perder a inconsistência.

$\mathrm{Na}$ eletromiografia de superfície, um fato que pode contribuir para essa variabilidade é o local onde o eletrodo é posicionado, como mencionado no estudo de Mesin et. al. (2009), que relata que há 3 estratégias para o posicionamento dos eletrodos, como no centro ou o mais próximo do ventre muscular, em algum local entre a zona de inervação e o tendão distal e no ponto motor. Neste estudo ele verificou que em 21 estudos, que analisavam o posicionamento dos eletrodos de superfície na eletromiografia, 4 não apresentaram sequer o local que foi posicionado. Os outros ficaram entre estas 3 estratégias.

Além disso, o estudo de Farina; Merletti; Enoka(2014), relata que outros fatores podem influenciar na variabilidade do sinal eletromiográfico, como a estrutura física do eletrodo, como a estrutura e a geometria, as propriedades anátomofisiológicas dos músculos e a tarefa realizada. Os resultados do presente estudo mostraram exatamente estas dificuldades, sendo principalmente a estrutura física dos músculos do antebraço.

Mesmo com o SENIAM realizando estudos multicêntricos, ainda não há um consenso em relação ao posicionamento dos eletrodos no antebraço, o que vai ao encontro com os estudos de Zakaria; Kramer; Harnburn, 1996.

Oskouei; Paulin; Carman (2013) revelaram que quanto maior a força de preensão maior a variabilidade, porém isto não afetou o valor de confiabilidade. Tal resultado, corrobora com este estudo, na qual foi avaliado $100 \%$ da CMV e os índices, intra-dias, se mostraram com valores de ICC maiores que 0,750, como preconizado por Fleiss (1986). 
Um dado importante encontrado no presente estudo, foi o baixo valor de confiabilidade, em alguns canais, em ambos os posicionamentos, quando foi realizado no grupo com indivíduos que apresentavam lesão, grupo pacientes.

Tal fato pode ser explicado, pelo membro avaliado estar acometido por uma lesão, o que faz com que a atividade elétrica esteja diminuída e assim afetando o sinal eletromiográfico. Isto acomete a parte sensório motora do indivíduo e assim a percepção do membro esteja afetado, acarretando em uma diminuição da efetividade da contração muscular (KARAGIANNOPOULOS, et. al., 2013).

O mesmo não pode se aferir pela diminuição da força, como foi verificado neste estudo na avaliação inicial, na qual todos os pacientes apresentaram um déficit de força em relação ao lado contra-lateral, pois no estudo de Oskouei; Paulin; Carman (2013) verificou-se que a confiabilidade a $5 \%$ da máxima contração voluntária apresentou excelentes resultados e que a retirada dos eletrodos e a recolocação não foi afetada.

O estudo de $\mathrm{Ng}$ et. al. (2003) relata que há dificuldade em se determinar o verdadeiro valor máximo da contração voluntária e isso apresenta uma grande imprecisão na medida e consequentemente variabilidade nos resultados do sinal de eletromiografia.

Porém, o presente estudo demonstrou uma confiabilidade alta mesmo sendo realizado com a máxima contração voluntária, como no estudo de Oskouei et. al. (2012), que apresentou valores de ICC maiores que 0.800 de correlação em todos os músculos analisados.

Quando analisamos os resultados obtidos dos eletrodos e comparando entre eles, percebe-se que os canais 4 e 5 , referentes aos músculos flexor ulnar do carpo e flexor superficial dos dedos, apresentaram os menores índices de correlação tanto no método de Cram; Durie como no de Mogk; Keir.

A concordância, por meio do gráfico de dispersão de Bland e Altman, do teste e re-teste dos dois métodos de posicionamento, verifica uma alta relação em ambos. Pode verificar que em todos os canais os valores ficam próximos da média, próximo do zero. Tal fato faz com que os dois métodos se tornem concordantes em sua realização.

No estudo de Bland; Altman (1999) demostraram que este método é mais fidedigno para a realização de estudos de confiabilidade, pois quando se trata de confiabilidade as ferramentas devem apresentar concordância entre os valores. 
$\mathrm{Na}$ qual demostra que o presente estudo, através das duas metodologias de posicionamento de eletrodo de superfície na eletromiografia, apresenta valores relevantes de concordância entre eles.

Todos os canais utilizados obtiveram resultados próximos da linha média, o que demonstra uma grande relação de concordância.

Quando comparamos os resultados obtidos pelo método estatístico de coeficiente de correlação intra-classe com os gráficos de Bland; Altman, os valores não são concordantes. Visto que no ICC os valores foram altos, com boa correlação, mas quando se olha os gráficos de Bland; Altman, pode se perceber que os valores estão dispersos, porém dentro do intervalo de confiança de $95 \%$, revelando uma correlação média.

O presente estudo verificou que o posicionamento dos eletrodos é parte fundamental na análise eletromiográfica. Sem um correto posicionamento ocorrerão interferências captadas de outros músculos e assim ocorrendo uma falha na aquisição.

Ainda não é possível realizar uma padronização para este posicionamento, como o grupo SENIAM realizou, pois como o antebraço apresenta músculos com ventres musculares pequenos e muito próximos, e com uma grande amplitude de movimento, sendo que os eletrodos estarão na superfície, qualquer movimentação a captação estará prejudicada.

Mas, com este estudo, mais um passo foi dado para a padronização, pois verificou que as metodologias são reprodutíveis e confiáveis.

Pela metodologia de Mogk; Keir, por ser circular e englobar toda a parte proximal do antebraço, na qual se apresentam a maioria dos ventres musculares, nas tarefas dinâmicas, os eletrodos conseguiriam captar os sinais eletromiográficos.

Neste sentido, ainda são necessários novos estudos que correlacionem as posturas do antebraço, as tarefas e a interferência no sinal captado, afim de apresentar uma padronização fidedigna, como há em outras regiões no corpo humano. 
CONCLUSÃO 


\section{Conclusão}

Os resultados do presente estudo demonstraram que o método de Cram e Durie, apresenta melhores valores de ICC do que de Mogk e Keir. Porém quando analisados pelos gráficos de Bland-Altman ambos apresentam alta concordancia. Mais estudos se fazem necessários para avaliar o melhor posicionamento em diferentes populações e quantificação da interferência do sinal eletromiográfico. 
REFERÊNCIAS 
ARAÚJO, R. C.; TUCCI, H. T.; ANDRADE, R.; MARTINS, J.; BEVILAQUA-GROSSI, D.; OLIVEIRA, A. S. Reliability of Electromyographic amplitude values of the upper limb muscles during closed kinetic chain exercises with stable and unstable surfaces. Journal of Electromyography and Kinesiology, vol. 19, p. 685-694, 2009.

BAO, S.; SILVERSTEIN, B.; Estimation of hand force in ergonomic job evaluations, Ergonomics, vol. 48, n.3, p. 288-301, 2005.

BLAND J. M.; ALTMAN D. G. Measuring agreement in method comparison studies.

Statistical Methods in Medical Research. vol. 8, n. 2, p. 135-60. 1999

CRAM, J.R.; DURIE, M. The History of Muscle Dysfunction and Surface Electromyography. In: CRISWELL, E. Cram's Introduction to Surface Electromyography. Sadbury:Jones and Bartlett Publishers, 2011. 175-190.

DE LUCA, C. J. The use of surfasse Electromyography in biomechanics. J Appl Biomech, v. 13, p. 135-163, 1997.

De VET, H. C. W.; TERWEE, C. B.; MOKKINK, L. B.; KNOL, D. L. Reliability. In: Measurement in Medicine: A Practical Guide. New York: Cambridge University Press. p. 96-145. 2011.

FAGARASANU, M.; KUMAR, S. Measurement instruments and data collection: a consideration of constructs and biases in ergonomics research. Int $\mathbf{J}$ Ind Ergon, vol. 30, p. 355-69, 2002.

FARINA, D.; MERLETTI, R.; STEGEMAN, D. F. Biophysics of the Generation of EMG Signals. In: MERLETTI, R.; PARKER, P. A. Electromyography: Physiology, Engineering, and Noninvasive Applications. New Jersey: IEEE, 2004. 91-97.

FARINA, D; MERLETTI, R.; ENOKA, R.M. The extraction of neural strategies from the surface EMG: an update. Journal of Applied Physiology. Vol. 117. 1215-1230, 2014.

FESS, E.E. Functional Tests. In: SKIRVEN, T. M. et al. (Ed.). Rehabilitation of the hand and upper extremity. 6th ed. Philadelphia :Mosby, 2011.cap 12, p.152-162. 
FINSEN, L.; SOGAARD, K.; GRAVEN-NIELSEN, T.; CHRISTENSEN, H. Activity Patterns of Wrist Extensor Muscles During Wrist Extensions and Desviations. Muscle Nerve, 31: 242-251, 2005.

FLEISS, J. L. Analysis of data from multiclinic trials. Control Clin Trials, vol. 7, n. 4, p. 267-275, 1986.

HAIG, A. J.; GOODMURPHY, C. W.; HARRIS, A. R.; RUIZ, A. P.; ETEMAD, J. The Accuracy of Needle Placement in Lower-Limb Muscles: A Blinded Study. Arch Phys Med Rehabil, v. 84, p. 877-882, 2003.

HERMENS, J. H.; FRERIKS, B.; DISSELHORST-KLUG, C.; RAU, G. Development of recommendations for SEMG sensors and sensor placement procedures. Journal of Electromyography and Kinesiology. V. 10, p. 361-374, 2000.

HERMSDÖRFER, J; LI, Y; RANDERATH, J; GOLDENBERG, G; EIDENMÜLLER, S. Anticipatory scaling of grip forces when lifting objects everyday life. Exp rain Res, 212: 19-31, 2011.

HINTERMEISTER, R. A.; LANGE, G. W.; SCHULTHEIS, J. M.; BEY, M.J.; HAWKINS, R. J. Electromyographic Activity and Applied Load During Shoulder Rehabilitation Exercises Using Elastic Resistance. Am J Sports Med, v. 26(2), p. 210-220, 1998.

IACONO, C. U. Test-retest reliability of static EMG scan configural profiling. Appl Psychophysiol Biofeedback, vol. 29, n. 1, p. 35-50, 2004.

JARUS, T.; POREMBA, R. Hand Function Evaluation: A Factor Analysis Study. The American Journal of Occupational Therapy, vol. 47(5): 439-443, 1993.

KARAGIANNOPOULOS, C.; SITLER, M.; MICHLOVITZ, S.; TIERNEY, R. $A$ descriptive study on wrist and hand sensori-motor impairment and function following distal radius fracture intervention. J Hand Ther, vol. 26, n. 3, p. 204-214, Jul-Sep, 2013.

LUNDBORG, G; ROSÉN, B. Hand Function after nerve repair. Acta Physiol, 189, 207-217, 2007. 
MACDERMID, J. C. Outcome Measurement in Upper Extremity Practice. In: SKIRVEN, T. M.; OSteRMAN, A. L.; FEDORCZYK, J. M.; AMADIO, P. C. Rehabilitation of the Hand and Upper Extremity. Philadelphia: Mosby, 2011. 194-205.

MELLSTRAND-NAVARRO, C.; PETTERSSON, H. J.; TORNQVIST, H.; PONZER, S.; The operative treatment of fractures of the distal radius is increasing: results from a nationwide Swedish study. Bone Joint J, vol. 96-B, n. 7, p. 963-999, Jul. 2014.

MESIN, L.; MERLETTI, R.; RAINOLDI, A. Surface EMG: The issue of electrode location. Journal of Electromyography and Kinesiology, vol. 19, p. 719-726, 2009.

MOGK, J. P. M.; KEIR, P. J. Crosstalk in surface electromyography of the proximal forearm during gripping tasks. Journal of Electromyography and Kinesiology, vol. 13, p. 63-71, 2003.

NEUMANN, D. Elbow and Forearm Complex. In: NEUMANN, D. Kynesiology of the Musculoskeletal System: Foundations for Physical Rehabilitation. Philadelphia: Mosby, 2002. 133-171.

NG, J. K.; PARNIANPOUR, M.; KIPPERS, V.; RICHARDSON, C. A. Reliability of electromyographic and torque measures during isometric axial rotation exertions of the trunk. Clin Neurophysiol, v.114, n. 12, p. 2355-2361, 2003.

OlLIVIER, K.; PORTERO, P.; MAISETTI, O.; HOGREL, J. Y.; Repeatability of surface EMG parameters at various isometric contraction levels and during fatigue using bipolar and Laplacian electrode configurations. J Electromyogr Kinesiol, vol. 15, n. 5, p. 466-473. 2005.

ONYEBEKE, L. C.; YOUNG, J. G.; TRUDEAU, M. B.; DENNERLEIN, J. T.; Effects of forearm and palm supports on the upper extremity during computer mouse use. Appl Ergon. Vol. 45, n. 3, p. 564-570, May. 2014.

OSKOUEI, A. H.; PAULIN, M. G.; CARMAN, A. B. Intra-session and inter-day reliability surface EMG during varying hand grip forces. Journal of Electromyography and Kinesiology, 2012.

JARUS, T.; POREMBA, R.; Hand function evaluation: a factor analysis study. Am J Occup Ther, v. 47, n. 5, p. 439-443, May. 1993. 
QUACH, J. H. Surface Electromyography: Use, Design \& Technological Overview. 2007.

ROSÉN, B.; LUNDBORG, G.; Enhanced sensory recovery after median nerve repair using cortical audio-tactile interaction. A randomized multicentre study. J Hand Surg Eur, vol. 32, n.1, p. 31-37, Feb. 2007.

RIORDAN, D.C. A Walk through the Anatomy of the Hand and Forearm. Journal of Hand Therapy, April-June, 1995.

SODEBERG, G. L.; KNUSTSON, L. M. A guide for use and interpretation of kinesiologic electromyographic data. Phys Ther, v.80 (5), p. 485-98, maio 2000.

STRICKLAND, J.W. Anatomy and Kinesiology of the Upper Extremity. In: FESS, E.E.; GETTLE, K; PHILIPS, C.; JANSON, R. Hand and Upper Extremity Splinting: Principles \& Methods. Philadelphia: Mosby, 2005. 47-85.

ZAKARIA, D.; KRAMER, J. F.; HARBURN, K. L. Reliability of non-normalized and normalized integrated EMG during maximal isometric contractions in females. Journal of Electromyography and Kinesiology. vol. 6, n², 129-135, 1996.

ZIMMERMAN, N.B. Clinical application of advances in elbow and forearm anatomy and biomechanics. Hand Clin, 18:1-19, 2002. 
APÊNDICE 


\section{Apêndice - A Termo de Consentimento Livre e Esclarecido}

Convidamos o (a) senhor (a) a participar do estudo intitulado "Análise da confiabilidade do posicionamento dos eletrodos na aquisição do sinal eletromiográfico dos músculos do antebraço" que será realizada na cidade de Ribeirão Preto - SP, tendo como pesquisadores responsáveis a Profa. Dra. Marisa de Cássia Registro Fonseca da Faculdade de Medicina de Ribeirão Preto USP e o mestrando Guilherme Tamanini da Faculdade de Medicina de Ribeirão Preto - USP.

Esclarecimento geral e objetivo do estudo: O presente estudo tem por objetivo verificar a confiabilidade do posicionamento dos eletrodos, na aquisição do sinal eletromiográfico de superfície, na tarefa de preensão palmar. Esta pesquisa é importante, pois poderá explicar por que alguns pacientes sentem dificuldade na realização de tarefas como segurar objetos.

Explicação do procedimento: As coletas ocorrerão em datas previamente agendadas entre o pesquisador e o $\mathrm{Sr}$ (a), sendo que a segunda e a terceira coletas deverão ser em no máximo 10 dias após a primeira coleta.

O Sr. (a) participará do estudo como voluntário. Inicialmente iremos realizar a tricotomia da pele, isto é a retirada dos pêlos do braço através do uso de uma Lâmina de Barbear e/ou aparelho próprio para tal procedimento. Após será realizado a limpeza da pele do Sr. (a) com álcool 70\% e também a esfoliação com uma lixa de unha de maneira leve e suave, isto serve para a retirada de células mortas que ficam na pele. Em seguida o Sr. (a) irá realizar movimentos de abrir e fechar os dedos e movimentação com o punho, para que o pesquisador possa verificar o local exato dos músculos do antebraço do Sr. (a), e assim marcá-los com uma caneta própria para tal. Serão colocados nos pontos marcados eletrodos passivos onde serão responsáveis pela captação do sinal. Este eletrodo serve somente de captação e o Sr. (a) não sentirá qualquer incômodo. Para a aquisição do sinal EMG o Sr. (a) irá realizar o movimento de aperto de mão em um aparelho que medirá a sua força.

Possíveis Benefícios: O (a) $\mathrm{Sr}$ (a) deve estar ciente de que não existem benefícios diretos à sua pessoa devido à participação nesta pesquisa e que os dados obtidos pelos responsáveis auxiliarão no maior conhecimento da ferramenta estudada.

Desconforto e Risco: O (a) Sr. (a) foi informado que este experimento poderá apresentar uma sensação de cansaço e/ou fadiga no braço e mão na qual será realizado o procedimento após a execução dos testes.

Liberdade de participação: A sua participação neste estudo é voluntária. É seu direito se recusar a participar ou mesmo interromper sua participação a qualquer momento, sem que isso cause qualquer penalidade ou prejuízo à sua pessoa.

Sigilo de identidade: As informações obtidas nesta pesquisa serão de maneira alguma associadas a sua identidade e não poderão ser consultadas por outras pessoas sem sua autorização oficial. Estas informações poderão ser utilizadas para fins estatísticos ou científicos, desde que fiquem guardados a sua identidade e seu anonimato. 
Os responsáveis pelo estudo se comprometem a explicar a necessidade da pesquisa, seus objetivos e procedimentos, bem como os benefícios e todos os riscos envolvidos. Além disso, os pesquisadores se prontificaram a responder todas as suas questões sobre o experimento e oferecer assistência a qualquer dano eventual decorrente da pesquisa, durante a após a sua realização. É seu dever manter uma cópia deste consentimento.

$\mathrm{Eu}$ portador no $\mathrm{RG} \mathrm{n}^{\circ}$

Residente à bairro

Cidade declaro que tenho anos de idade e que concordo em participar, voluntariamente, na pesquisa conduzida pelos alunos responsáveis e por seu (sua) respectivo (a) orientador (a).

Assinatura do voluntário:

Ribeirão Preto, de de 20

Para questões relacionadas a este estudo, contate:

Profa. Dra. Marisa de Cássia Registro Fonseca e Guilherme Tamanini Curso de Fisioterapia - Faculdade de Medicina de Ribeirão Preto - FMRP-USP Fone: (0XX16) 988264188 - Email: marisa@fmrp.usp.br / guilhermetamanini@usp.br

Guilherme Tamanini

Pesquisador Responsável

Profa. Dr. Marisa de Cássia Registro Fonseca

Orientadora Responsável 
Anexo - A

Carta de aprovação do Comitê de Ética e Pesquisa do Hospital das Clínicas da Faculdade de Medicina de Ribeirão Preto da Universidade de São Paulo.

HOSPITAL DAS CLÍNICAS DA FACULDADE DE MEDICINA DE RIBEIRÃO PRETO DA UNIVERSIDADE DE SÄO PAULO

Ribeirão Preto, 05 de fevereiro de 2013

Oficio $\mathrm{n}^{\circ} 368 / 2014$

$\mathrm{CEP} / \mathrm{MGV}$

PROCESSO HCRP n ${ }^{\circ} 13049 / 2013$

Prezados Pesquisadores,

O trabalho intitulado "ANÁLISE DA CONFIABILIDADE DO POSICIONAMENTO DOS ELETRODOS NA AQUISIÇÃO DO SINAL ELETROMIOGRÁFICO DOS MÚSCULOS DO ANTEBRAÇO", foi analisado pelo Comitê de Ética em Pesquisa, em sua $381^{a}$ Reunião Ordinária realizada em 03/02/2014, e enquadrado na categoria: APROVADO, bem como o Termo de Consentimento Livre e Esclarecido Segunda Versão - 06/01/2014.

Este Comitê segue integralmente a Conferência Internacional de Harmonização de Boas Práticas Clínicas (IGH-GCP), bem como a Resolução no 196/96 CNS/ MS.

Lembramos que devem ser apresentados a este CEP, o Relatório Parcial e o Relatório Final da pesquisa. De acordo com Carta Circular $n^{\circ}$ 003/2011/CONEP/CNS, datada de 21/03/2011, o sujeito de pesquisa ou seu representante, quando for o caso, deverá rubricar todas as folhas do Termo de Consentimento Livre e Esclarecido - TCLE - apondo sua assinatura na última do referido Termo; o pesquisador responsável deverá da mesma forma, rubricar todas as folhas do Termo de Consentimento Livre e Esclarecido - TCLE - apondo sua assinatura na última página do referido Termo.

Atenciosamente.

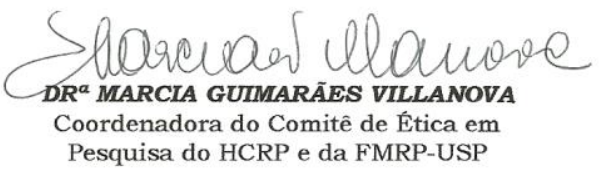

Ilustríssimos Senhores

GUILHERME TAMANINI

PROF ${ }^{a}$ DR ${ }^{a}$ MARISA DE CÁSSIA REGISTRO FONSECA (Orientadora)

Depto. de Biomecânica, Medicina e Reabilitaçāo do Aparelho Locomotor

\begin{tabular}{|c|c|}
\hline Campus Universitário - Monte Alegre & Comitê de Ética em Pesquisa do HCRP e FMRP-USP \\
\hline 14048-900 Ribeirāo Preto SP & $\begin{array}{r}\text { FWA-00002733; IRB-00002186 e Registro PB/CONEP n⿳ } 5440 \\
\text { (016) } 3602-2228 \\
\text { cep@hcrp.usp.br }\end{array}$ \\
\hline
\end{tabular}

